\title{
A One-Pot Multicomponent Coupling Reaction for the Stereocontrolled Synthesis of (Z)-Trisubstituted Allylic Alcohols
}

\author{
Young K. Chen and Patrick J. Walsh ${ }^{*}$ \\ Contribution from the P. Roy and Diane T. Vagelos Laboratories, University of \\ Pennsylvania, Department of Chemistry 231 South 34th Street, Philadelphia, \\ Pennsylvania 19104-6323. \\ pwalsh@sas.upenn.edu
}

\section{Table of Contents}

Table of Contents $\quad$ S1

$\begin{array}{ll}\text { General Methods } & \text { S2 }\end{array}$

$\begin{array}{ll}\text { Supporting Information } & \text { S2 }\end{array}$

$\begin{array}{ll}\text { References } & \text { S14 }\end{array}$

NMR $\left({ }^{1} \mathrm{H}\right.$ and $\left.{ }^{13} \mathrm{C}\right) \quad$ S15 


\section{Supporting Information}

General Methods. All reactions involving dialkylzinc, trialkylborane, and $\mathrm{Br}_{2} \mathrm{BH}$ were carried out in a Vacuum Atmospheres dry box or under nitrogen using standard Schlenk techniques. Diethylborane in toluene was generated according to a procedure by Knochel $^{1}$ and kept in Schlenk storage vessel capped with a Teflon ${ }^{\circledR}$ stopcock. Neat $\mathrm{Br}_{2} \mathrm{BH}$ were purchased from Aldrich Chemical Company, and made into a $1.0 \mathrm{M}$ solution in dry toluene and kept in Schlenk storage vessel capped with a Teflon ${ }^{\circledR}$ stopcork. NMR spectra were obtained on a Brüker $500 \mathrm{MHz}$ Fourier transform spectrometer at the University of Pennsylvania NMR facility. ${ }^{1} \mathrm{H}$ NMR spectra were referenced to tetramethylsilane in $\mathrm{CDCl}_{3} ;{ }^{13} \mathrm{C}\left\{{ }^{1} \mathrm{H}\right\}$ NMR spectra were referenced to residual solvent. Dry toluene and hexanes were obtained by passage through activated alumina columns. All reagents were purchased from Aldrich Chemical Company or GFS Chemical. Bromoalkynes were made according to known procedures. ${ }^{2}$ All nonsolid aldehydes were distilled prior to use and stored in an Schlenk storage vessel capped with a Teflon ${ }^{\circledR}$ stopcock. Paraformaldehyde was dried over $\mathrm{P}_{2} \mathrm{O}_{5}$ at $0.100 \mathrm{~mm} \mathrm{Hg}$ vacuum for 24 hours.

\section{General Procedure A}

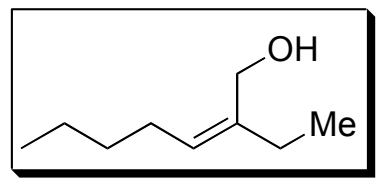

(Z)-Ethyl-hept-2-en-ol: To a dry and thoroughly purged $\left(\mathrm{N}_{2}\right)$

$10 \mathrm{~mL}$ Schlenk flask capped with a rubber septum was charged

with 1-bromo-1-hexyne $(193 \mu \mathrm{L}, 1.5 \mathrm{mmol})$, and $0.5 \mathrm{~mL}$ toluene. The solution was cooled to $0{ }^{\circ} \mathrm{C}$ followed by dropwise addition of $\mathrm{Et}_{2} \mathrm{BH}(1.5 \mathrm{~mL}, 1.5 \mathrm{mmol}, 1.0 \mathrm{M})$. The reaction was stirred for $5 \mathrm{~min}$, warmed to ambient temperature, and stirred for $15 \mathrm{~min}$. 
The clear solution was cooled to $-78{ }^{\circ} \mathrm{C}$ and $\mathrm{Et}_{2} \mathrm{Zn}$ in toluene $(3.6 \mathrm{~mL}, 3.6 \mathrm{mmol}, 1.0 \mathrm{M})$ was added. After stirring at this temperature for $15 \mathrm{~min}$, the reaction was warmed to $0{ }^{\circ} \mathrm{C}$ and paraformaldehyde $(90 \mathrm{mg}, 3.0 \mathrm{mmol})$ was added. The reaction was allowed to warm to ambient temperature and stirred for $14 \mathrm{hrs}$ before the reaction was quenched with saturated $\mathrm{NH}_{4} \mathrm{Cl}(4 \mathrm{~mL}) .2 \mathrm{~N} \mathrm{HCl}(1.0 \mathrm{~mL})$ was added to the heterogeneous suspension followed by $5 \mathrm{~mL}$ of ethyl acetate. The clear biphasic layers were partitioned and the aqueous layer was repeatedly extracted with ethyl acetate $(3 \mathrm{X} 10 \mathrm{~mL})$. The combined organic layer was washed with saturated $\mathrm{NaHCO}_{3}$ followed by saturated $\mathrm{NaCl}$, dried over anhydrous $\mathrm{MgSO}_{4}$, and concentrated. The residue was purified by column chromatography (5-10\% ethyl acetate in hexanes) to yield the desired product as colorless oil (149 mg, $70 \%$ based on 1-bromo-1-hexyne). ${ }^{1} \mathrm{H}$ NMR ( $\left.\mathrm{CDCl}_{3}, 500 \mathrm{MHz}\right): \delta$ $0.89(\mathrm{t}, 3 \mathrm{H}, J=7.1 \mathrm{~Hz}), 1.0(\mathrm{t}, 3 \mathrm{H}, J=7.4 \mathrm{~Hz}), 1.30-1.34(\mathrm{~m}, 4 \mathrm{H}), 2.08(\mathrm{~m}, 2 \mathrm{H}), 2.14(\mathrm{q}$, $2 \mathrm{H}, J=7.4 \mathrm{~Hz}), 4.15(\mathrm{~d}, 2 \mathrm{H}, J=4.8 \mathrm{~Hz})$, and $5.31(\mathrm{t}, 1 \mathrm{H}, J=7.4 \mathrm{~Hz}) \mathrm{ppm} .{ }^{13} \mathrm{C}\left\{{ }^{1} \mathrm{H}\right\}$ NMR $\left(\mathrm{CDCl}_{3}, 125 \mathrm{MHz}\right): \delta 13.3,14.3,22.7,27.6,28.2,32.7,60.7,128.0$, and $140.3 \mathrm{ppm}$. IR (neat): 3327, $1460 \mathrm{~cm}^{-1}$. HRMS-CI $m / z 125.1328\left[(\mathrm{M}-\mathrm{OH})^{+}\right.$; calc for $\mathrm{C}_{9} \mathrm{H}_{17}$ : 125.1330].

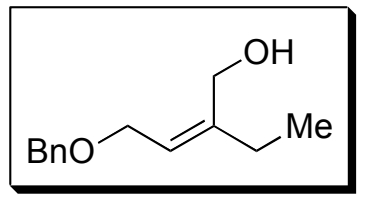

(Z)-4-Benzyloxy-2-ethyl-but-2-en-1-ol: According to general procedure A. 3-bromo-(1-benzyloxy)-prop-2-yne (328 mg, 1.5 $\mathrm{mmol}), \mathrm{Et}_{2} \mathrm{BH}(1.5 \mathrm{~mL}, 1.5 \mathrm{mmol}, 1.0 \mathrm{M}), \mathrm{Et}_{2} \mathrm{Zn}(3.6 \mathrm{~mL}, 3.6$ mmol, $1.0 \mathrm{M}$ ), and paraformaldehyde (90 mg, $3.0 \mathrm{mmol})$. Afforded $189 \mathrm{mg}$ (61 \% yield) of viscous oil after column chromatography (10-15\%, ethyl acetate in hexanes). ${ }^{1} \mathrm{H}$ NMR $\left(\mathrm{CDCl}_{3}, 500 \mathrm{MHz}\right): \delta 1.06(\mathrm{t}, 3 \mathrm{H}, J=7.4 \mathrm{~Hz}), 1.95(\mathrm{~s}, 1 \mathrm{H},-\mathrm{OH}), 2.18(\mathrm{q}, 2 \mathrm{H}, J=7.4$ 
$\mathrm{Hz}), 4.10(\mathrm{~m}, 4 \mathrm{H}), 4.53(\mathrm{~s}, 2 \mathrm{H}), 5.57(\mathrm{t}, 1 \mathrm{H}, J=6.7 \mathrm{~Hz})$ and $7.28-7.35(\mathrm{~m}, 5 \mathrm{H}) \mathrm{ppm}$.

${ }^{13} \mathrm{C}\left\{{ }^{1} \mathrm{H}\right\} \mathrm{NMR}\left(\mathrm{CDCl}_{3}, 125 \mathrm{MHz}\right): \delta 12.8,28.8,61.4,66.3,72.9,122.8,128.2,128.3$, 128.9, 138.4, and $147.0 \mathrm{ppm}$. IR (neat): $3364,1589,1470$, and $1427 \mathrm{~cm}^{-1}$. HRMS-CI $\mathrm{m} / z$ $189.1275\left[(\mathrm{M}-\mathrm{OH})^{+}\right.$; calc for $\left.\mathrm{C}_{13} \mathrm{H}_{17} \mathrm{O}_{1}: 189.1279\right]$.

\section{General Procedure B}

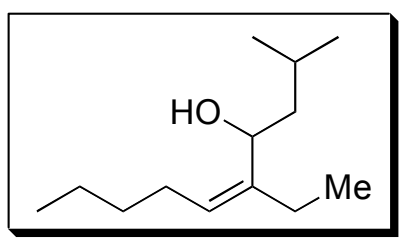

(Z)-5-Ethyl-2-methyl-dec-5-en-4-ol: To a dry and thoroughly purged $\left(\mathrm{N}_{2}\right) 10 \mathrm{~mL}$ Schlenk flask capped with a rubber septum was charged with 1-bromo-1-hexyne (193 $\mu \mathrm{L}, 1.5 \mathrm{mmol})$, and $0.5 \mathrm{~mL}$ of toluene. The solution was cooled to $0{ }^{\circ} \mathrm{C}$ followed by dropwise addition of $\mathrm{Et}_{2} \mathrm{BH}(1.5 \mathrm{~mL}, 1.5 \mathrm{mmol}, 1.0 \mathrm{M})$. The reaction was stirred for $5 \mathrm{~min}$ then warmed to ambient temperature over 15 min. The clear solution was cooled to $-78{ }^{\circ} \mathrm{C}$ and $\mathrm{Et}_{2} \mathrm{Zn}$ in toluene $(3.6 \mathrm{~mL}, 3.6 \mathrm{mmol}, 1.0 \mathrm{M})$ was added dropwise and stirred at this temperature for 15 min before warming to $0{ }^{\circ} \mathrm{C}$. Under a steady flow of $\mathrm{N}_{2}$, the rubber septum was replaced with a glass stopper coated with silicon grease and high vacuum was gradually applied. After most of the volatile contents are evacuated, the resulting vinylzinc reagent was redissolved in $1 \mathrm{~mL}$ of toluene and isovaleraldehyde $(108 \mu \mathrm{L}, 1.0 \mathrm{mmol})$ was added at $0{ }^{\circ} \mathrm{C}$. The reaction was allowed to warm to ambient temperature and stirred for $14 \mathrm{hrs}$ before dropwise addition of $\mathrm{NH}_{4} \mathrm{Cl}(4 \mathrm{~mL})$, followed by $2 \mathrm{~N} \mathrm{HCl}(1.0 \mathrm{~mL})$, and $5 \mathrm{~mL}$ of ethyl acetate. The clear biphasic layers were partition and the aqueous layer was extracted successively with ethyl acetate $(3 \times 10 \mathrm{~mL})$. The combined organic layer was washed with saturated $\mathrm{NaHCO}_{3}$ followed by saturated $\mathrm{NaCl}$, dried over anhydrous $\mathrm{MgSO}_{4}$, and concentrated. The residue was purified by column chromatography $(2.5-5 \%$ ethyl 
acetate in hexanes) to yield the desired product as colorless oil $(121 \mathrm{mg}, 61 \%$ based on aldehyde). ${ }^{1} \mathrm{H}$ NMR ( $\left.\mathrm{CDCl}_{3}, 500 \mathrm{MHz}\right): \delta$ 0.88-0.95 (m, 9H), 1.04 (t, 3H, $J=7.3 \mathrm{~Hz}$ ), $1.25(\mathrm{~s},-\mathrm{OH}, 1 \mathrm{H}), 1.26-1.39(\mathrm{~m}, 5 \mathrm{H}), 1.51-1.68(\mathrm{~m}, 2 \mathrm{H}), 1.95-2.10(\mathrm{~m}, 4 \mathrm{H}), 4.70(\mathrm{~m}, 1 \mathrm{H})$, and $5.20(\mathrm{t}, 1 \mathrm{H}, J=7.3 \mathrm{~Hz}) \mathrm{ppm} .{ }^{13} \mathrm{C}\left\{{ }^{1} \mathrm{H}\right\}$ NMR $\left(\mathrm{CDCl}_{3}, 125 \mathrm{MHz}\right): \delta 13.7,14.3,22.6$, 22.7, 22.9, 23.6, 25.1, 27.2, 32.7, 44.8, 68.7, 126.0, and 142.6 ppm. IR (neat): 3405, 1666, and $1457 \mathrm{~cm}^{-1}$. HRMS-CI $m / z 198.1989$ [M $\mathrm{M}^{+}$; calc for $\left.\mathrm{C}_{13} \mathrm{H}_{26} \mathrm{O}: 189.1984\right]$.

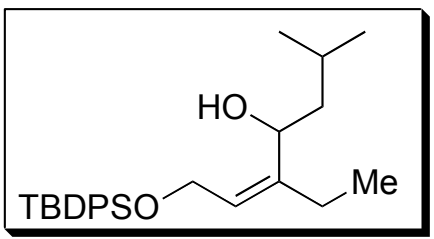

\section{(Z)-5-(tert-Butyl-diphenyl-silyloxy)-3-ethyl-6-methyl-hept-}

2-en-4-ol: According to general procedure B. (3-bromo-prop2-ynyloxy)-tert-butyldiphenylsilane (428 $\mathrm{mg}, 0.75 \mathrm{mmol})$, $\mathrm{Et}_{2} \mathrm{BH}(0.75 \mathrm{~mL}, 0.75 \mathrm{mmol}, 1.0 \mathrm{M}), \mathrm{Et}_{2} \mathrm{Zn}(1.8 \mathrm{~mL}, 1.8 \mathrm{mmol}, 1.0 \mathrm{M})$, and isovaleraldehyde $(54 \mu \mathrm{L}, 0.5 \mathrm{mmol})$. Afforded $145 \mathrm{mg}$ ( $71 \%$ yield) of clear oil after column chromatography (2.5-5\%, ethyl acetate in hexanes). ${ }^{1} \mathrm{H} \mathrm{NMR}\left(\mathrm{CDCl}_{3}, 500 \mathrm{MHz}\right)$ : $\delta 0.81$ (overlapping doublets, $6 \mathrm{H}, J=7.6, J=7.6 \mathrm{~Hz}$ ), 1.02 (t, $3 \mathrm{H}, J=7.4 \mathrm{~Hz}$ ), 1.04 (s, 9H), $1.16(\mathrm{~m}, 1 \mathrm{H}), 1.37(\mathrm{~s},-\mathrm{OH}, 1 \mathrm{H}), 1.45(\mathrm{~m}, \mathrm{H}), 1.55(\mathrm{~m}, 1 \mathrm{H}), 1.91-2.12(\mathrm{~m}, 2 \mathrm{H}), 4.22$ (m, 1H), $4.32(\mathrm{~m}, 2 \mathrm{H}), 5.39(\mathrm{t}, 1 \mathrm{H}, J=7.2 \mathrm{~Hz}), 7.34-7.47(\mathrm{~m}, 6 \mathrm{H})$, and $7.70(\mathrm{~m}, 4 \mathrm{H}) \mathrm{ppm}$. ${ }^{13} \mathrm{C}\left\{{ }^{1} \mathrm{H}\right\} \mathrm{NMR}\left(\mathrm{CDCl}_{3}, 125 \mathrm{MHz}\right): \delta 13.2,17.4,22.4,23.53,23.54,24.9,27.1,44.3,60.3$, $68.9,124.3,127.9,128.0,130.0,130.1,134.0,135.1,135.9,136.0$, and $145.7 \mathrm{ppm}$. IR (neat): $3431,1735,1589,1471$, and $1427 \mathrm{~cm}^{-1}$. HRMS-ES $m / z 433.2539\left[(\mathrm{M}+\mathrm{Na})^{+}\right.$; calc for $\mathrm{C}_{26} \mathrm{H}_{38} \mathrm{O}_{2} \mathrm{NaSi}$ : 433.2556]. 


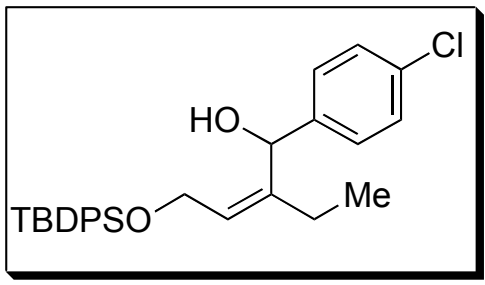

(Z)-4-(tert-Butyl-diphenyl-silyloxy)-1-(4-chlorophenyl)-

2-ethyl-but-2-en-1-ol: According to general procedure B. (3-Bromo-prop-2-ynyloxy)-tert-butyldiphenylsilane (428 $\mathrm{mg}, 0.75 \mathrm{mmol}), \mathrm{Et}_{2} \mathrm{BH}(0.75 \mathrm{~mL}, 0.75 \mathrm{mmol}, 1.0 \mathrm{M})$,

$\mathrm{Et}_{2} \mathrm{Zn}(1.8 \mathrm{~mL}, 1.8 \mathrm{mmol}, 1.0 \mathrm{M})$, and $p$-chlorobenzaldehyde $(70 \mathrm{mg}, 0.5 \mathrm{mmol})$. Afforded $195 \mathrm{mg}$ (84\% yield) of clear oil after column chromatography (2.5-5\%, ethyl acetate in hexanes). ${ }^{1} \mathrm{H}$ NMR $\left(\mathrm{CDCl}_{3}, 500 \mathrm{MHz}\right):{ }^{1} \mathrm{H} \mathrm{NMR}\left(\mathrm{CDCl}_{3}, 500 \mathrm{MHz}\right): \delta 0.91$ (t, $3 \mathrm{H}, J=7.4 \mathrm{~Hz}), 1.05(\mathrm{~s}, 9 \mathrm{H}), 1.76(\mathrm{~m}, 1 \mathrm{H}), 2.0(\mathrm{~m}, 1 \mathrm{H}), 2.07(\mathrm{~s},-\mathrm{OH}, 1 \mathrm{H}), 4.33-4.43(\mathrm{~m}$, 2H), $5.41(\mathrm{~s}, 1 \mathrm{H}), 5.59(\mathrm{t}, 1 \mathrm{H}, J=7.2 \mathrm{~Hz}), 7.18-7.25(\mathrm{~m}, 4 \mathrm{H}), 7.38-7.45(\mathrm{~m}, 6 \mathrm{H})$, and 7.68-7.71 (m, 4H) ppm. ${ }^{13} \mathrm{C}\left\{{ }^{1} \mathrm{H}\right\} \mathrm{NMR}\left(\mathrm{CDCl}_{3}, 125 \mathrm{MHz}\right): \delta 12.9,19.3,24.0,26.7,27.0$, $60.4,71.4,125.6,127.5,128.0128 .1,128.5,130.1,132.9,133.7,133.8,135.0,135.9$, 136.0, 140.8, and $145.2 \mathrm{ppm}$. IR (neat): $3424,1661,1589,1489$, and $1471 \mathrm{~cm}^{-1}$. HRMSES $m / z 487.1857$ [(M+Na) ${ }^{+}$; calc for $\left.\mathrm{C}_{28} \mathrm{H}_{33} \mathrm{ClO}_{2} \mathrm{NaSi}: 487.1836\right]$.

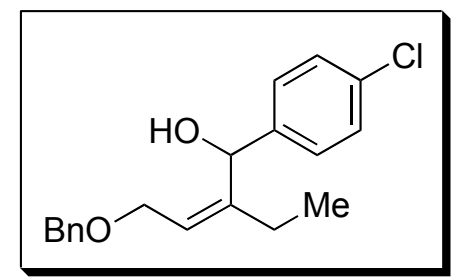

(Z)-4-Benzyloxy-1-(4-chlorophenyl)-2-ethyl-but-2-en-1-ol. According to procedure B. 3-bromo-(1-benzyloxy)-2propyne (328 mg, $1.5 \mathrm{mmol}), \mathrm{Et}_{2} \mathrm{BH}(1.5 \mathrm{~mL}, 1.5 \mathrm{mmol}, 1.0$ M), $\mathrm{Et}_{2} \mathrm{Zn}(3.6 \mathrm{~mL}, 3.6 \mathrm{mmol}, 1.0 \mathrm{M})$, and $p$-chlorobenzaldehyde (140.1 mg, $\left.1.0 \mathrm{mmol}\right)$. Afforded $272 \mathrm{mg}$ (86 \% yield) of viscous oil after column chromatography (5-10\%, ethyl acetate in hexanes). ${ }^{1} \mathrm{H}$ NMR $\left(\mathrm{CDCl}_{3}, 500 \mathrm{MHz}\right): \delta 1.0$ (t, 3H, $\left.J=7.4 \mathrm{~Hz}\right), 1.90$ (m, 1H), $2.15(\mathrm{~m}, 1 \mathrm{H}), 2.60(\mathrm{~d}, 1 \mathrm{H}, J=4.2 \mathrm{~Hz}), 4.17(\mathrm{~m}, 1 \mathrm{H}), 4.26(\mathrm{~m}, 1 \mathrm{H}), 4.58(\mathrm{~d}, 2 \mathrm{H}, J=$ $3.4 \mathrm{~Hz}), 5.61(\mathrm{~d}, 1 \mathrm{H}, J=4.2 \mathrm{~Hz}), 5.69(\mathrm{t}, 1 \mathrm{H}, J=4.2 \mathrm{~Hz})$ and 7.31-7.40 (m, 9H) ppm. ${ }^{13} \mathrm{C}\left\{{ }^{1} \mathrm{H}\right\}$ NMR $\left(\mathrm{CDCl}_{3}, 125 \mathrm{MHz}\right): \delta 12.9,24.7,66.2,71.8,73.2,122.8,127.7,128.3$, 128.4, 128.7, 128.9, 133.2, 138.2, 141.1, and $148.2 \mathrm{ppm}$. IR (neat): 3404, 1665, 1600, 
1586, 1496, and $1454 \mathrm{~cm}^{-1}$. HRMS-ES $\mathrm{m} / z$ 339.1128 [(M+Na) ${ }^{+}$; calc for $\mathrm{C}_{19} \mathrm{H}_{21} \mathrm{ClO}_{2} \mathrm{Na}$ : 339.1128].

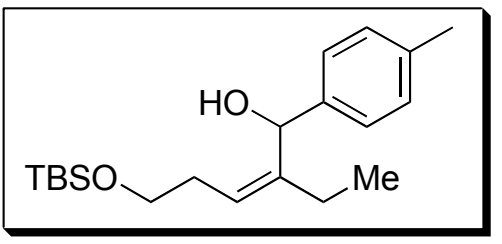

\section{(Z)-5-(tert-Butyl-dimethyl-silyloxy)-2-ethyl-1-p-tolyl-} pent-2-en-1-ol: According to general procedure B. (4Bromo-but-3-ynyloxy)-tert-butyldimethylsilane (197 mg, $0.75 \mathrm{mmol}), \mathrm{Et}_{2} \mathrm{BH}(0.75 \mathrm{~mL}, 0.75 \mathrm{mmol}, 1.0 \mathrm{M}), \mathrm{Et}_{2} \mathrm{Zn}(1.8 \mathrm{~mL}, 1.8 \mathrm{mmol}, 1.0 \mathrm{M})$, and p-tolualdehyde (60 $\mu \mathrm{L}, 0.5 \mathrm{mmol})$. Afforded $137 \mathrm{mg}$ (82\% yield) of clear oil after column chromatography (2.5-5\%, ethyl acetate in hexanes). ${ }^{1} \mathrm{H} \mathrm{NMR}\left(\mathrm{CDCl}_{3}, 500 \mathrm{MHz}\right)$ : $\delta 0.02(\mathrm{~s}, 6 \mathrm{H}), 0.93(\mathrm{~s}, 9 \mathrm{H}), 0.96(\mathrm{t}, 3 \mathrm{H}, J=7.2 \mathrm{~Hz}), 1.85(\mathrm{~m}, 1 \mathrm{H}), 2.04(\mathrm{~m}, 1 \mathrm{H}), 2.34(\mathrm{~s}$, $3 \mathrm{H}), 2.43-2.75(\mathrm{~m}, 2 \mathrm{H}), 2.75(\mathrm{~d}, 1 \mathrm{H},-\mathrm{OH}, J=3.3 \mathrm{~Hz}), 3.70(\mathrm{td}, 2 \mathrm{H}, J=6.2, J=2.0 \mathrm{~Hz})$ $5.36(\mathrm{t}, 1 \mathrm{H}, J=7.9 \mathrm{~Hz}), 5.61(\mathrm{~d}, 1 \mathrm{H}, J=3.0 \mathrm{~Hz}), 7.14(\mathrm{~d}, 2 \mathrm{H}, J=7.9 \mathrm{~Hz})$, and $7.28(\mathrm{~d}$, $2 \mathrm{H}, J=7.9 \mathrm{~Hz}) \mathrm{ppm} .{ }^{13} \mathrm{C}\left\{{ }^{1} \mathrm{H}\right\} \mathrm{NMR}\left(\mathrm{CDCl}_{3}, 125 \mathrm{MHz}\right): \delta-5.0,13.2,18.8,21.4,25.4$, 26.3, 31.4, 63.0, 72.2, 123.0, 126.2, 129.0, 136.6, 140.2, and 145.6 ppm. IR (neat): 3420 , 1511, and $1462 \mathrm{~cm}^{-1}$. HRMS-ES $\mathrm{m} / z$ 357.2215 $\left[(\mathrm{M}+\mathrm{Na})^{+}\right.$; calc for $\mathrm{C}_{20} \mathrm{H}_{34} \mathrm{O}_{2} \mathrm{NaSi}$ : 357.2226].

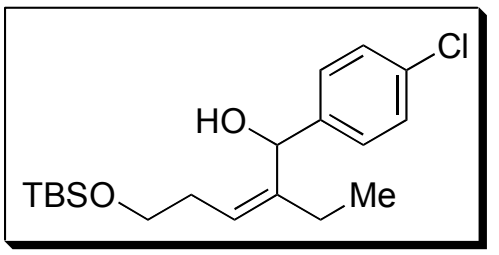

(Z)-5-(tert-Butyl-dimethyl-silyloxy)-1-(4-chlorophenyl)-2-ethyl-pent-2-en-1-ol: According to general procedure $\quad$ B. (4-Bromo-but-3-ynyloxy)-tertbutyldimethylsilane (197 mg, $0.75 \mathrm{mmol}), \mathrm{Et}_{2} \mathrm{BH}(0.75 \mathrm{~mL}, 0.75 \mathrm{mmol}, 1.0 \mathrm{M}), \mathrm{Et}_{2} \mathrm{Zn}$ (1.8 mL, $1.8 \mathrm{mmol}, 1.0 \mathrm{M})$, and p-chlorobenzaldehyde (70 mg, $0.5 \mathrm{mmol})$. Afforded 149 mg (84\% yield) of clear oil after column chromatography $(2.5-5 \%$, ethyl acetate in hexanes). ${ }^{1} \mathrm{H} \mathrm{NMR}\left(\mathrm{CDCl}_{3}, 500 \mathrm{MHz}\right): \delta 0.01$ (s, 6H), $0.91(\mathrm{~s}, 9 \mathrm{H}), 0.97$ (t, 3H, $J=7.4$ 
$\mathrm{Hz}), 1.81(\mathrm{~m}, 1 \mathrm{H}), 2.0(\mathrm{~m}, 1 \mathrm{H}), 2.44-2.49(\mathrm{~m}, 2 \mathrm{H}), 3.00(\mathrm{~s}, 1 \mathrm{H},-\mathrm{OH}), 3.71(\mathrm{~m}, 2 \mathrm{H}), 5.38$ (t, $1 \mathrm{H}, J=8.0 \mathrm{~Hz}), 5.58(\mathrm{~s}, 1 \mathrm{H}), 7.27-7.35(\mathrm{~m}, 4 \mathrm{H}) \mathrm{ppm} .{ }^{13} \mathrm{C}\left\{{ }^{1} \mathrm{H}\right\} \mathrm{NMR}\left(\mathrm{CDCl}_{3}, 125\right.$ MHz): $\delta-5.1,13.2,18.8,25.7,26.3,31.3,62.9,71.6,123.9,127.7,128.4,132.7,141.7$, and $145.5 \mathrm{ppm}$. IR (neat): 3386, 1489, and $1470 \mathrm{~cm}^{-1}$. HRMS-ES $\mathrm{m} / z 377.1672$ $\left[(\mathrm{M}+\mathrm{Na})^{+}\right.$; calc. for $\mathrm{C}_{19} \mathrm{H}_{31} \mathrm{ClO}_{2} \mathrm{NaSi}$ : 377.1680$]$.

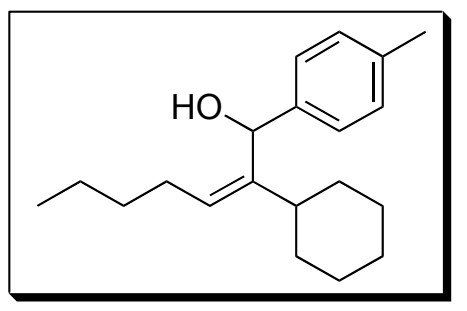

(Z)-2-Cyclohexyl-p-tolyl-hept-2-en-1-ol: 1-Bromo-1hexyne (197 mg, $0.75 \mathrm{mmol}), \mathrm{Cy}_{2} \mathrm{BH}$ (133 mg, $0.75 \mathrm{mmol}$ ), $\mathrm{Et}_{2} \mathrm{Zn}(1.8 \mathrm{~mL}, 1.8 \mathrm{mmol}, 1.0 \mathrm{M})$, and $p$-tolualdehyde (60 $60 \mu \mathrm{L}, 0.5 \mathrm{mmol})$. Afforded $86 \mathrm{mg}$ (60\% yield) of clear oil after column chromatography (1-2.5\%, ethyl acetate in hexanes). ${ }^{1} \mathrm{H}$ NMR $\left(\mathrm{CDCl}_{3}, 500\right.$ MHz): $\delta 0.90(\mathrm{t}, 3 \mathrm{H}, J=7.2 \mathrm{~Hz}), 1.0-1.45(\mathrm{~m}, 10 \mathrm{H}), 1.53-1.77(\mathrm{~m}, 5 \mathrm{H}), 1.94(\mathrm{~m}, 1 \mathrm{H})$, $2.20(\mathrm{~m}, 2 \mathrm{H}), 2.33(\mathrm{~s}, 3 \mathrm{H}) 5.40(\mathrm{t}, 1 \mathrm{H}, J=7.4 \mathrm{~Hz}), 5.72(\mathrm{~s}, 1 \mathrm{H}), 7.12(\mathrm{~d}, 2 \mathrm{H}, J=8.0 \mathrm{~Hz})$ and $7.25(\mathrm{~d}, 2 \mathrm{H}, J=7.3 \mathrm{~Hz}) \mathrm{ppm} .{ }^{13} \mathrm{C}\left\{{ }^{1} \mathrm{H}\right\} \mathrm{NMR}\left(\mathrm{CDCl}_{3}, 125 \mathrm{MHz}\right): \delta 14.3,21.3,22.7$, $26.5,27.3,27.7,32.7,34.8,35.7,39.3,71.9,125.9,127.4,129.0,136.5,140.1$, and 146.6 ppm. IR (neat): $3463,1703,1659,1605,1511$, and $1447 \mathrm{~cm}^{-1}$. HRMS-ES $m / z 285.2203$ $\left[(\mathrm{M}-\mathrm{H})^{+}\right.$; calc for $\left.\mathrm{C}_{20} \mathrm{H}_{29} \mathrm{O}: 285.2218\right]$.

(Z)-2-Cyclohexyl-1-(2-methoxy-phenyl)-hept-2-en-1-ol: 1-Bromo-1-hexyne (197 mg, $0.75 \mathrm{mmol}), \mathrm{Cy}_{2} \mathrm{BH}(133 \mathrm{mg}, 0.75 \mathrm{mmol}), \mathrm{Et}_{2} \mathrm{Zn}(1.8 \mathrm{~mL}, 1.8 \mathrm{mmol}, 1.0 \mathrm{M})$, and $o-$ methoxybenzaldehyde (68 mg, $0.5 \mathrm{mmol})$. Afforded $95 \mathrm{mg}$ ( $63 \%$ yield) of clear oil after column chromatography (1-2.5 \%, ethyl acetate in hexanes). ${ }^{1} \mathrm{H} \mathrm{NMR}\left(\mathrm{CDCl}_{3}, 500 \mathrm{MHz}\right)$ : $\delta 0.89(\mathrm{t}, 3 \mathrm{H}, J=7.2 \mathrm{~Hz}), 1.0-1.40(\mathrm{~m}, 11 \mathrm{H}), 1.58(\mathrm{~m}, 2 \mathrm{H}), 1.73(\mathrm{~m}, 1 \mathrm{H}), 1.80(\mathrm{~m}, 1 \mathrm{H})$, $2.0(\mathrm{~m}, 1 \mathrm{H}), 2.16(\mathrm{~m}, 2 \mathrm{H}), 2.50(\mathrm{~s}, 1 \mathrm{H},-\mathrm{OH}), 3.83(\mathrm{~s}, 3 \mathrm{H}), 5.39(\mathrm{t}, 1 \mathrm{H}, J=7.2 \mathrm{~Hz}), 5.87$ 


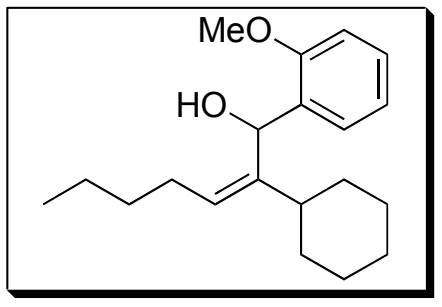

(s, 1H), $6.84(\mathrm{~d}, 1 \mathrm{H}, J=8.2 \mathrm{~Hz}), 6.93(\mathrm{t}, 1 \mathrm{H}, J=7.5 \mathrm{~Hz})$

$7.22(\mathrm{t}, 1 \mathrm{H}, J=6.5 \mathrm{~Hz})$, and $7.40(\mathrm{~d}, 1 \mathrm{H}, J=6.6 \mathrm{~Hz}) \mathrm{ppm}$.

${ }^{13} \mathrm{C}\left\{{ }^{1} \mathrm{H}\right\}$ NMR $\left(\mathrm{CDCl}_{3}, 125 \mathrm{MHz}\right): \delta 14.5,22.9,26.8,27.5$, $27.6,28.0,32.7,34.8,35.2,40.2,55.7,69.2,110.6,120.8$, 127.7, 127.8, 128.5, 131.2, 144.6, and 157.4 ppm. IR (neat): 3433, 1702, 1600, 1489, 1464, and $1377 \mathrm{~cm}^{-1}$. HRMS-ES $m / z$ 285.2211 [(M-OH $)^{+}$; calc for $\left.\mathrm{C}_{20} \mathrm{H}_{29} \mathrm{O}_{1}: 285.2218\right]$.

\section{General Procedure C}

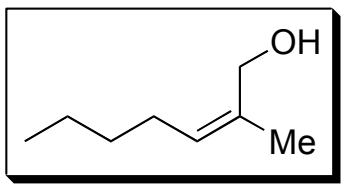

(Z)-Methyl-hept-2-en-1-ol: To a dry and thoroughly purged $\left(\mathrm{N}_{2}\right)$ $10 \mathrm{~mL}$ Schlenk flask charged with 1-bromo-1-hexyne (1.5 mmol) and $0.5 \mathrm{~mL}$ of toluene was added $1.5 \mathrm{~mL}$ of $\mathrm{Br}_{2} \mathrm{BH}(1.0 \mathrm{M}$ in toluene). The reaction was warmed to $70{ }^{\circ} \mathrm{C}$ and kept at this temperature for 1 hour. The solution was cooled to $-78{ }^{\circ} \mathrm{C}$ and $\mathrm{Me}_{2} \mathrm{Zn}$ in toluene $(3.0 \mathrm{~mL}, 6.0 \mathrm{mmol}, 2.0 \mathrm{M})$ was added dropwise and stirred at this temperature for 15 min then warmed to $0{ }^{\circ} \mathrm{C}$ and paraformaldehyde $(90 \mathrm{mg}, 3.0 \mathrm{mmol})$ was added. The reaction was allowed to warm to ambient temperature and stirred for 14 hrs. The reaction was quenched by dropwise addition of $\mathrm{NH}_{4} \mathrm{Cl}$, initially under a flow of $\mathrm{N}_{2}$, followed by the $2 \mathrm{~N} \mathrm{HCl}(1.0 \mathrm{~mL})$, and 5 $\mathrm{mL}$ of ethyl acetate. The clear biphasic layers were partitioned and the aqueous layer was successively extracted with ethyl acetate $(3 \mathrm{X} 10 \mathrm{~mL})$. The combined organic layer was washed with saturated $\mathrm{NaHCO}_{3}$ followed by saturated $\mathrm{NaCl}$, dried over anhydrous $\mathrm{MgSO}_{4}$ and concentrated. The residue was purified by column chromatography (10-15 $\%$ diethyl ether in pentane) to yield the desired product as colorless low boiling liquid 
(144 mg, $75 \%$ yield based on 1-bromo-1-hexyne). All spectroscopic data are identical to literature values. ${ }^{3,4}$

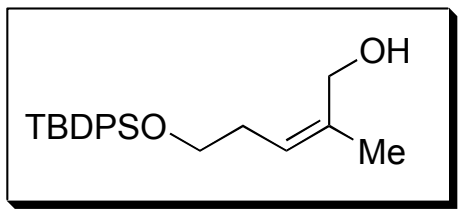

\section{(Z)-5-(tert-Butyl-diphenyl-silyloxy)-2-methyl-pent-2-en-}

1-ol: According to general procedure C. (4-Bromo-but-3ynyloxy)-tert-butyldiphenylsilane (580 $\mathrm{mg}, 1.5 \mathrm{mmol})$, $\mathrm{Br}_{2} \mathrm{BH}(1.5 \mathrm{~mL}, 1.5 \mathrm{mmol}, 1.0 \mathrm{M}), \mathrm{Me}_{2} \mathrm{Zn}(3.0 \mathrm{~mL}, 6.0 \mathrm{mmol}, 2.0 \mathrm{M})$, and paraformaldehyde (90 mg, $3.0 \mathrm{mmol})$. Afforded $409 \mathrm{mg}$ (77\% yield) of viscous oil after column chromatography (10-15\%, ethyl acetate in hexanes). ${ }^{1} \mathrm{H}$ NMR $\left(\mathrm{CDCl}_{3}, 500\right.$ MHz): $\delta 1.08(\mathrm{~s}, 9 \mathrm{H}), 1.73(\mathrm{~s}, 1 \mathrm{H},-\mathrm{OH}), 1.85(\mathrm{~s}, 3 \mathrm{H}), 2.37$ (q, 2H, $J=6.6 \mathrm{~Hz}), 3.67(\mathrm{t}$, $2 \mathrm{H}, J=6.2 \mathrm{~Hz}), 4.10(\mathrm{~d}, 2 \mathrm{H}, J=5.4 \mathrm{~Hz}), 5.33(\mathrm{t}, 1 \mathrm{H}, J=7.7 \mathrm{~Hz}), 7.40-7.48(\mathrm{~m}, 6 \mathrm{H})$, and 7.7 (m, 4H) ppm. ${ }^{13} \mathrm{C}\left\{{ }^{1} \mathrm{H}\right\}$ NMR $\left(\mathrm{CDCl}_{3}, 125 \mathrm{MHz}\right): \delta$ 19.6, 22.2, 27.3, 31.4, 62.2, 64.0, 125.1, 128.0, 130.1, 134.0, 136.0, and $137.2 \mathrm{ppm}$. IR (neat): 3342, 1658, 1589, 1472, 1428, 1389, and $1361 \mathrm{~cm}^{-1}$. HRMS-ES $m / z 377.1895\left[(\mathrm{M}+\mathrm{Na})^{+}\right.$; calc for $\mathrm{C}_{22} \mathrm{H}_{30} \mathrm{O}_{2} \mathrm{NaSi}$ : 377.1913].

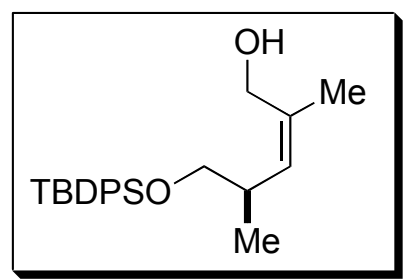

(Z,R)-5-(tert-Butyl-diphenyl-silyloxy)-2,4-dimethyl-pent-2-

en-1-ol: According to general procedure C. (4-Bromo-2methyl-but-3-ynyloxy)-tert-butyldiphenylsilane (602 mg, 1.5 $\mathrm{mmol}), \mathrm{Br}_{2} \mathrm{BH}(1.5 \mathrm{~mL}, 1.5 \mathrm{mmol}, 1.0 \mathrm{M}), \mathrm{Me}_{2} \mathrm{Zn}(3.0 \mathrm{~mL}$, $6.0 \mathrm{mmol}, 2.0 \mathrm{M})$, and paraformaldehyde $(90 \mathrm{mg}, 3.0 \mathrm{mmol})$. Afforded $268 \mathrm{mg}(73 \%$ yield) of viscous oil after column chromatography (10-15\%, ethyl acetate in hexanes). $[\alpha]^{20}{ }_{\mathrm{D}}=-8.0\left(c=0.53, \mathrm{CHCl}_{3}\right){ }^{1} \mathrm{H} \mathrm{NMR}\left(\mathrm{CDCl}_{3}, 500 \mathrm{MHz}\right): \delta 0.89(\mathrm{~d}, 3 \mathrm{H}, J=6.7 \mathrm{~Hz})$, 
$1.04(\mathrm{~s}, 9 \mathrm{H}), 1.81(\mathrm{~d}, 3 \mathrm{H}, J=1.5 \mathrm{~Hz}), 1.90(\mathrm{~m}, 1 \mathrm{H},-\mathrm{OH}), 2.77(\mathrm{~m}, 1 \mathrm{H}), 3.30(\mathrm{dd}, 1 \mathrm{H}, J$ $=8.75, J=8.14 \mathrm{~Hz}), 3.48(\mathrm{dd}, 1 \mathrm{H}, J=9.3, J=5.7 \mathrm{~Hz}), 3.94(\mathrm{dd}, 1 \mathrm{H}, J=12.0, J=7.2$ Hz), 4.12-4.18 (dd, $1 \mathrm{H}, J=12.0, J=4.7 \mathrm{~Hz}), 5.03(\mathrm{~d}, 1 \mathrm{H}, 9.9 \mathrm{~Hz}), 7.37-7.43(\mathrm{~m}, 6 \mathrm{H})$, and $7.6(\mathrm{~m}, 4 \mathrm{H}) \mathrm{ppm} .{ }^{13} \mathrm{C}\left\{{ }^{1} \mathrm{H}\right\}$ NMR $\left(\mathrm{CDCl}_{3}, 125 \mathrm{MHz}\right): \delta 17.8,19.1,22.3,27.3,35.4$, $62.6,69.3,128.1,130.1,132.2,133.9,136.0$, and $136.3 \mathrm{ppm}$. IR (neat): 3372, 1589, 1472, 1428, 1389, and $1361 \mathrm{~cm}^{-1}$. HRMS-ES $\mathrm{m} / \mathrm{z} 369.2233\left[\mathrm{M}^{+}\right.$; calc for $\mathrm{C}_{23} \mathrm{H}_{32} \mathrm{O}_{2} \mathrm{Si}$ : 369.2250]. All Spectroscopic data are identical to literature values. ${ }^{5,6}$ Both $(R)-3$ and $(S)$ 3 were converted to the corresponding $(R)$-Mosher ester derivatives and found to be diastereomerically pure.

\section{General Procedure D}

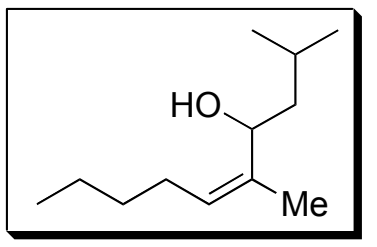

(Z)-2,5-Dimethyl-dec-5-en-4-ol: To a dry and thoroughly purged $\left(\mathrm{N}_{2}\right) 10 \mathrm{~mL}$ Schlenk flask capped with a rubber septum was charged with 1-bromo-1-hexyne $(193 \mu \mathrm{L}, 1.5 \mathrm{mmol})$ and 0.5 $\mathrm{mL}$ of toluene. $\mathrm{Br}_{2} \mathrm{BH}(1.5 \mathrm{~mL}, 1.5 \mathrm{mmol}, 1.0 \mathrm{M}$ in toluene) was added at ambient temperature. The reaction was warmed to $70{ }^{\circ} \mathrm{C}$. After 1 hour, the reaction was cooled to $-78^{\circ} \mathrm{C}$ and $\mathrm{Me}_{2} \mathrm{Zn}$ in toluene $(3.0 \mathrm{~mL}, 6.0 \mathrm{mmol}, 2.0 \mathrm{M})$ was added dropwise and stirred at this temperature for $15 \mathrm{~min}$ then warmed to $0{ }^{\circ} \mathrm{C}$. Under a steady flow of $\mathrm{N}_{2}$, the rubber septum was replaced with a glass stopper coated with silicon grease and high vacuum was gradually applied. After most of the volatile contents are evacuated, the resulting vinylzinc reagent was redissolved in $1 \mathrm{~mL}$ of toluene and isovaleraldehyde $(108 \mu \mathrm{L}, 1.0$ mmol) was added. The reaction was allowed to warm to ambient temperature and stirred for $14 \mathrm{hrs}$. The reaction was quenched by dropwise addition of $\mathrm{NH}_{4} \mathrm{Cl}$, followed by the 2 $\mathrm{N} \mathrm{HCl}(1.0 \mathrm{~mL})$, and $5 \mathrm{~mL}$ of ethyl acetate. The clear biphasic layers were partitioned 
and the aqueous layer was successively extracted with ethyl acetate $(3 \mathrm{X} 10 \mathrm{~mL})$. The combined organic layer was washed with saturated $\mathrm{NaHCO}_{3}$ followed by saturated $\mathrm{NaCl}$, dried over anhydrous $\mathrm{MgSO}_{4}$ and concentrated. The residue was purified by column chromatography (2.5-5\% ethyl acetate in hexanes) to yield the desired product as colorless oil (169 mg, $92 \%$ based on aldehyde). ${ }^{1} \mathrm{H}$ NMR $\left(\mathrm{CDCl}_{3}, 500 \mathrm{MHz}\right): \delta 0.88$ $0.95(\mathrm{~m}, 9 \mathrm{H}), 1.20-1.36(\mathrm{~m}, 6 \mathrm{H}$, and $-\mathrm{OH}), 1.55(\mathrm{~m}, 1 \mathrm{H}), 1.61(\mathrm{~m}, 1 \mathrm{H}), 1.68(\mathrm{~d}, 3 \mathrm{H}, J=$ $4.2 \mathrm{~Hz}), 1.95-2.10(\mathrm{~m}, 2 \mathrm{H}), 4.65(\mathrm{dd}, 1 \mathrm{H}, J=7.9, J=5.9 \mathrm{~Hz}), 5.23(\mathrm{t}, 1 \mathrm{H}, J=7.3 \mathrm{~Hz})$ ppm. ${ }^{13} \mathrm{C}\left\{{ }^{1} \mathrm{H}\right\}$ NMR $\left(\mathrm{CDCl}_{3}, 125 \mathrm{MHz}\right): \delta 14.4,17.8,22.8,22.9,23.7,25.2,27.4,32.7$, 44.5, 68.2, 128.3, and $137.3 \mathrm{ppm}$. IR (neat): 3405,1588 , and $1489 \mathrm{~cm}^{-1}$. HRMS-CI $\mathrm{m} / \mathrm{z}$ 184.1819 $\left[\mathrm{M}^{+}\right.$; calc for $\left.\mathrm{C}_{12} \mathrm{H}_{24} \mathrm{O}: 184.1827\right]$.

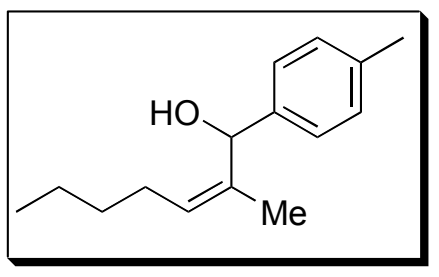

(Z)-2-Methyl-1-p-tolyl-hept-2-en-1-ol: According to general procedure D. 1-Bromo-1-hexyne ( $97 \mu \mathrm{L}, 0.75 \mathrm{mmol}), \mathrm{Br}_{2} \mathrm{BH}$ (0.75 mL, $0.75 \mathrm{mmol}, 1.0 \mathrm{M}), \mathrm{Me}_{2} \mathrm{Zn}(1.5 \mathrm{~mL}, 3.0 \mathrm{mmol}, 2.0$ $\mathrm{M})$, and $p$-tolualdehyde $(60 \mu \mathrm{L}, 0.5 \mathrm{mmol})$. Afforded $77 \mathrm{mg}$ ( $71 \%$ yield) of clear oil after column chromatography (2.5-5\%, ethyl acetate in hexanes). $\left.{ }^{1} \mathrm{H} \mathrm{NMR} \mathrm{(CDCl}, 500 \mathrm{MHz}\right): \delta 0.92(\mathrm{t}, 3 \mathrm{H}, J=7.6 \mathrm{~Hz}), 1.02$ (t, 3H, $\left.J=7.4 \mathrm{~Hz}\right), 1.04$ (s, 9H), $1.16(\mathrm{~m}, 1 \mathrm{H}), 1.37(\mathrm{~s},-\mathrm{OH}, 1 \mathrm{H}), 1.45(\mathrm{~m}, \mathrm{H}), 1.55(\mathrm{~m}, 1 \mathrm{H}), 1.91-2.12(\mathrm{~m}, 2 \mathrm{H}), 4.22$ (m, 1H), $4.32(\mathrm{~m}, 2 \mathrm{H}), 5.39(\mathrm{t}, 1 \mathrm{H}, J=7.2 \mathrm{~Hz}), 7.34-7.47(\mathrm{~m}, 6 \mathrm{H})$, and $7.70(\mathrm{~m}, 4 \mathrm{H}) \mathrm{ppm}$. ${ }^{13} \mathrm{C}\left\{{ }^{1} \mathrm{H}\right\}$ NMR $\left(\mathrm{CDCl}_{3}, 125 \mathrm{MHz}\right): \delta 13.7,14.3,22.6,22.7,22.9,23.6,25.1,27.2,32.7$, 44.8, 68.7, 126.0, and $142.6 \mathrm{ppm}$. IR (neat): 3379, 1511, 1455, and $1377 \mathrm{~cm}^{-1}$. HRMS-CI $m / z 218.1667\left[\mathrm{M}^{+}\right.$; calc for $\left.\mathrm{C}_{15} \mathrm{H}_{22} \mathrm{O}: 218.1671\right]$. 


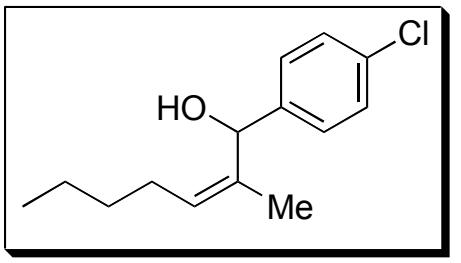

(Z)-1-(4-Choro-phenyl)-2-methyl-hept-2-en-1-ol:

According to general procedure D. 1-Bromo-1-hexyne (97 $\mu \mathrm{L}, 0.75 \mathrm{mmol}), \mathrm{Br}_{2} \mathrm{BH}(0.75 \mathrm{~mL}, 0.75 \mathrm{mmol}, 1.0 \mathrm{M})$, $\mathrm{Me}_{2} \mathrm{Zn}(1.5 \mathrm{~mL}, 3.0 \mathrm{mmol}, 2.0 \mathrm{M})$, and $p$-chlorobenzaldehyde $(70 \mathrm{mg}, 0.5 \mathrm{mmol})$. Afforded $83 \mathrm{mg}$ (70\% yield) of clear oil after column chromatography (2.5-5\% ethyl acetate in hexanes). ${ }^{1} \mathrm{H}$ NMR ( $\left.\mathrm{CDCl}_{3}, 500 \mathrm{MHz}\right): \delta 0.94$ (t, 3H, $\left.J=7.2 \mathrm{~Hz}\right), 1.37-1.43$ (m, 4H), $1.55(\mathrm{~s}, 3 \mathrm{H}), 1.73(\mathrm{~s},-\mathrm{OH}, 1 \mathrm{H}), 2.12-2.29(\mathrm{~m}, 2), 5.40(\mathrm{t}, 1 \mathrm{H}, J=7.2 \mathrm{~Hz}), 5.74$ (s, 1H), and $7.30(\mathrm{~s}, 4 \mathrm{H}) \mathrm{ppm} .{ }^{13} \mathrm{C}\left\{{ }^{1} \mathrm{H}\right\} \mathrm{NMR}\left(\mathrm{CDCl}_{3}, 125 \mathrm{MHz}\right): \delta 14.2,17.6,22.7,27.7$, $32.5,70.7,127.3,128.6,129.8,132.9,136.0$, and $141.2 \mathrm{ppm}$. IR (neat): 3369,1596 , and $1489 \mathrm{~cm}^{-1}$. HRMS-CI $\mathrm{m} / z 238.1117$ [ $\mathrm{M}^{+}$; calc for $\left.\mathrm{C}_{14} \mathrm{H}_{19} \mathrm{ClO}: 238.1124\right]$.

(1) Langer, F.; Schwink, L.; Devasagayaraj, A.; Chavant, P.-Y.; Knochel, P. Journal of Organic Chemistry 1996, 61, 8229-8243.

(2) Leroy, J. Organic Syntheses 1997, 74, 212-216.

(3) Rapoport, H.; Bhalerao, U. T. Journal of the American Chemical Society 1971, 93, 4835-4840.

(4) Lis, L.; Koltun, E. S.; Liu, H.-w.; Kass, S. R. Journal of the American Chemical Society 2002, 124, 1276-1287. 
(5) Yokokawa, F.; Asano, T.; Shioiri, T. Organic Letters 2000, 2, 4169-4172.

(6) Yokokawa, F.; Asano, T.; Shioiri, T. Tetrahedron 2001, 57, 6311-6327. 

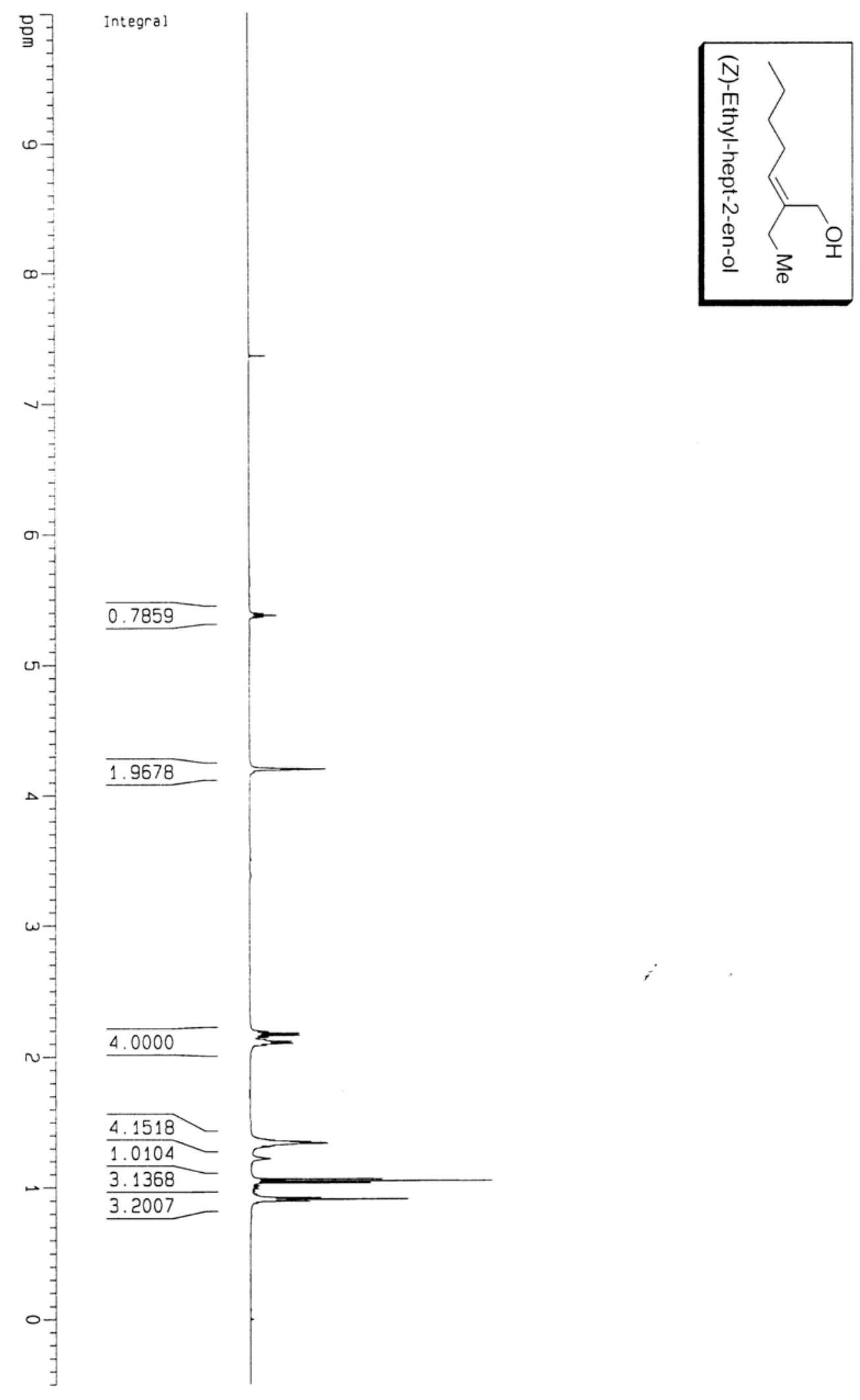


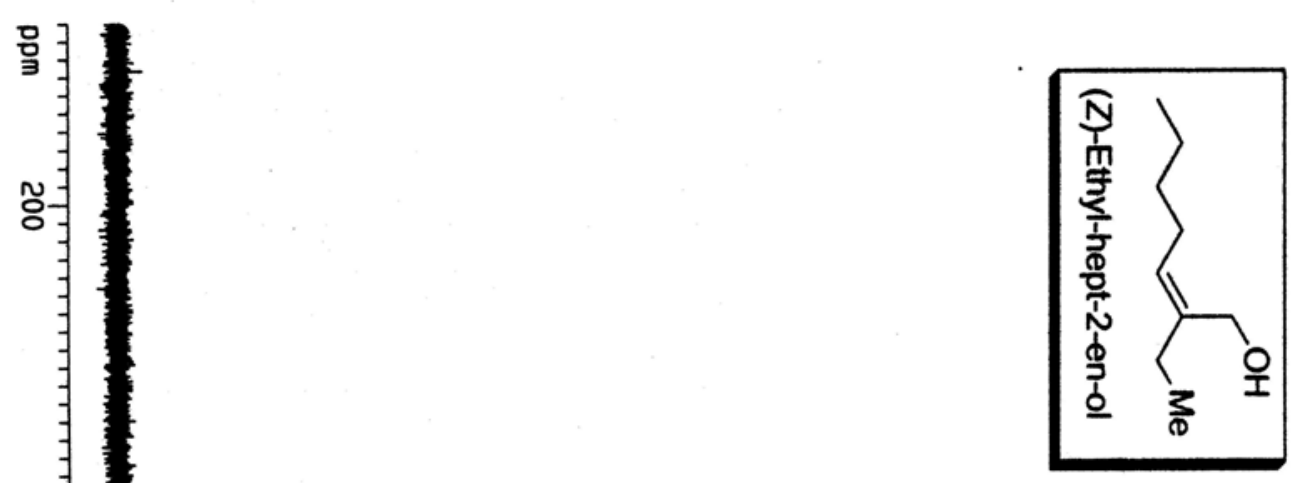

영-

븡-

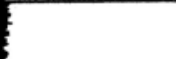

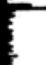

1

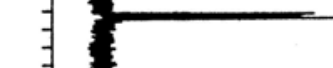

H

뎡-

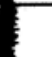



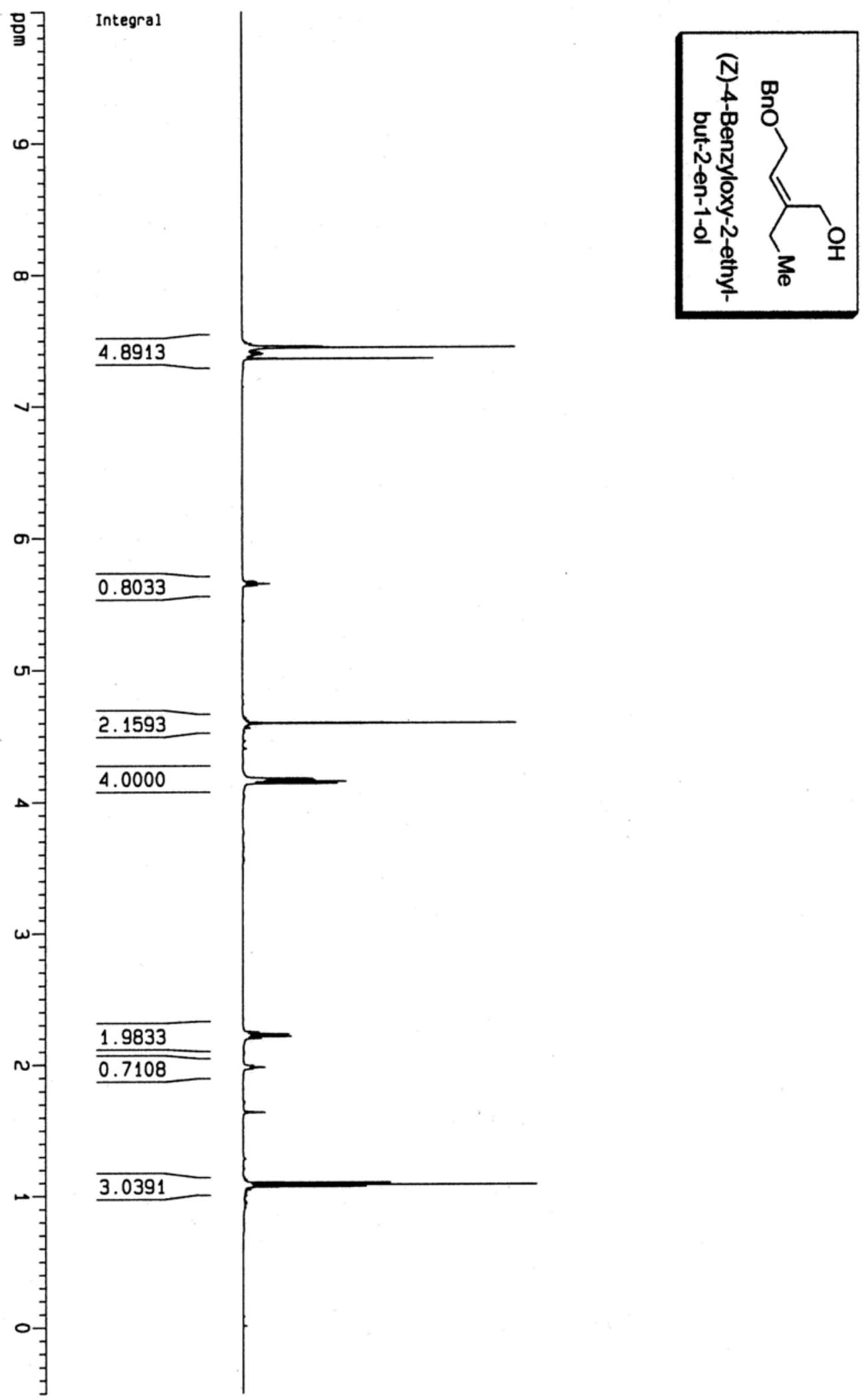

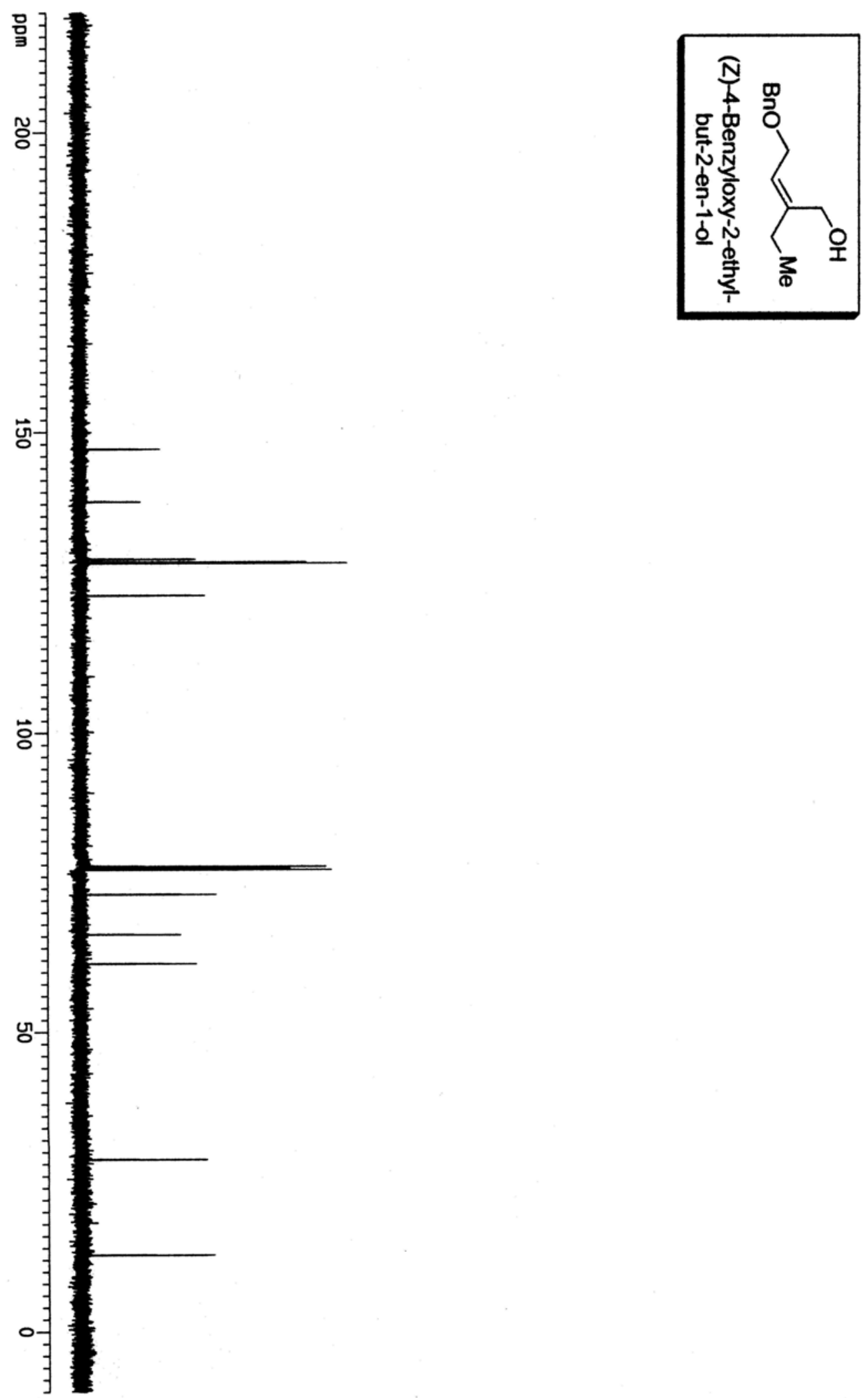

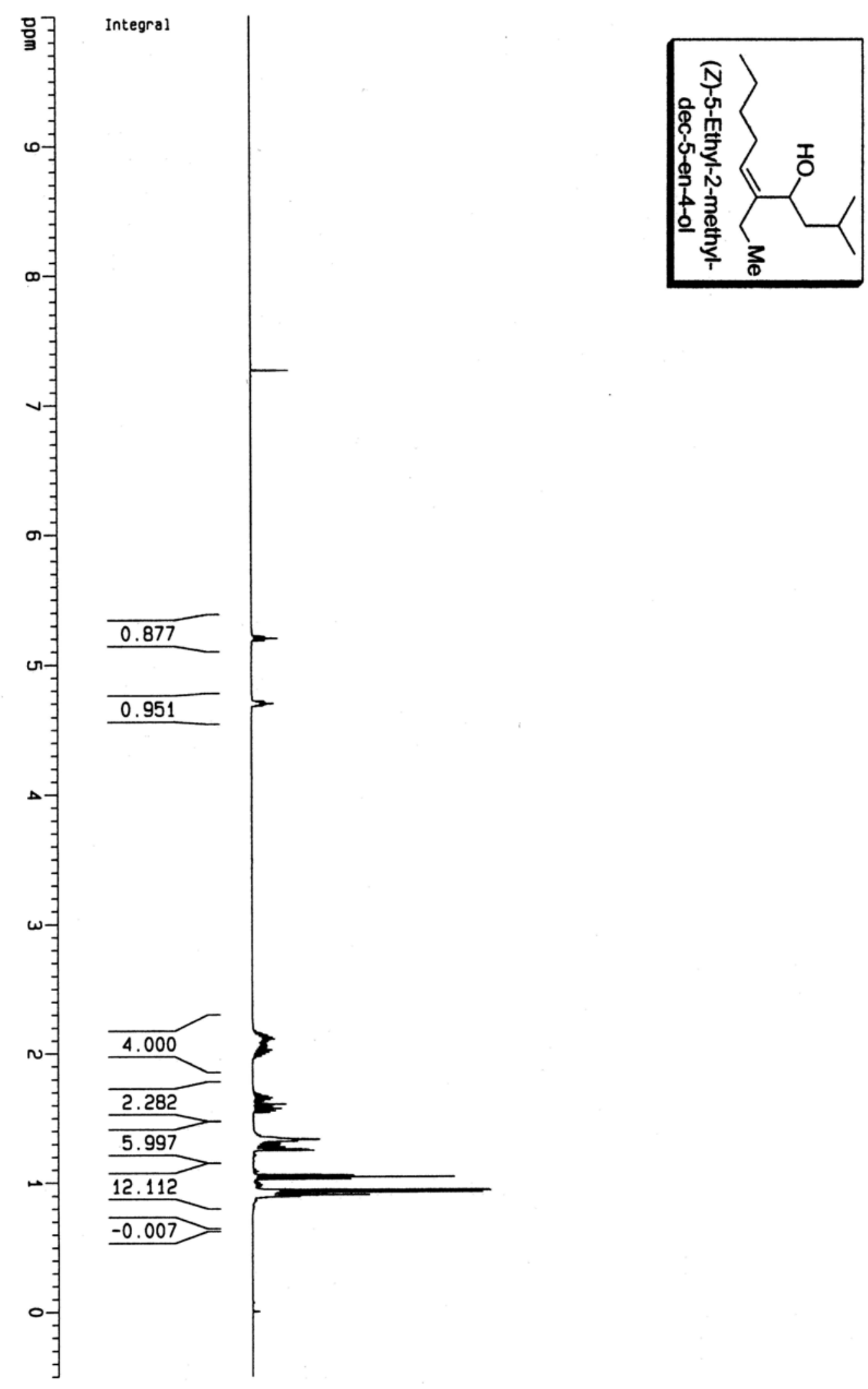


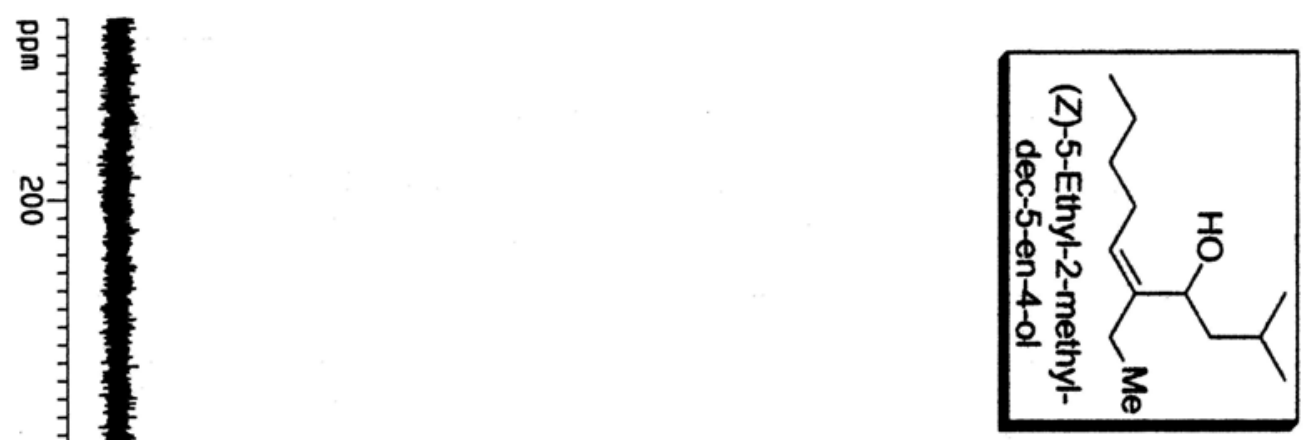




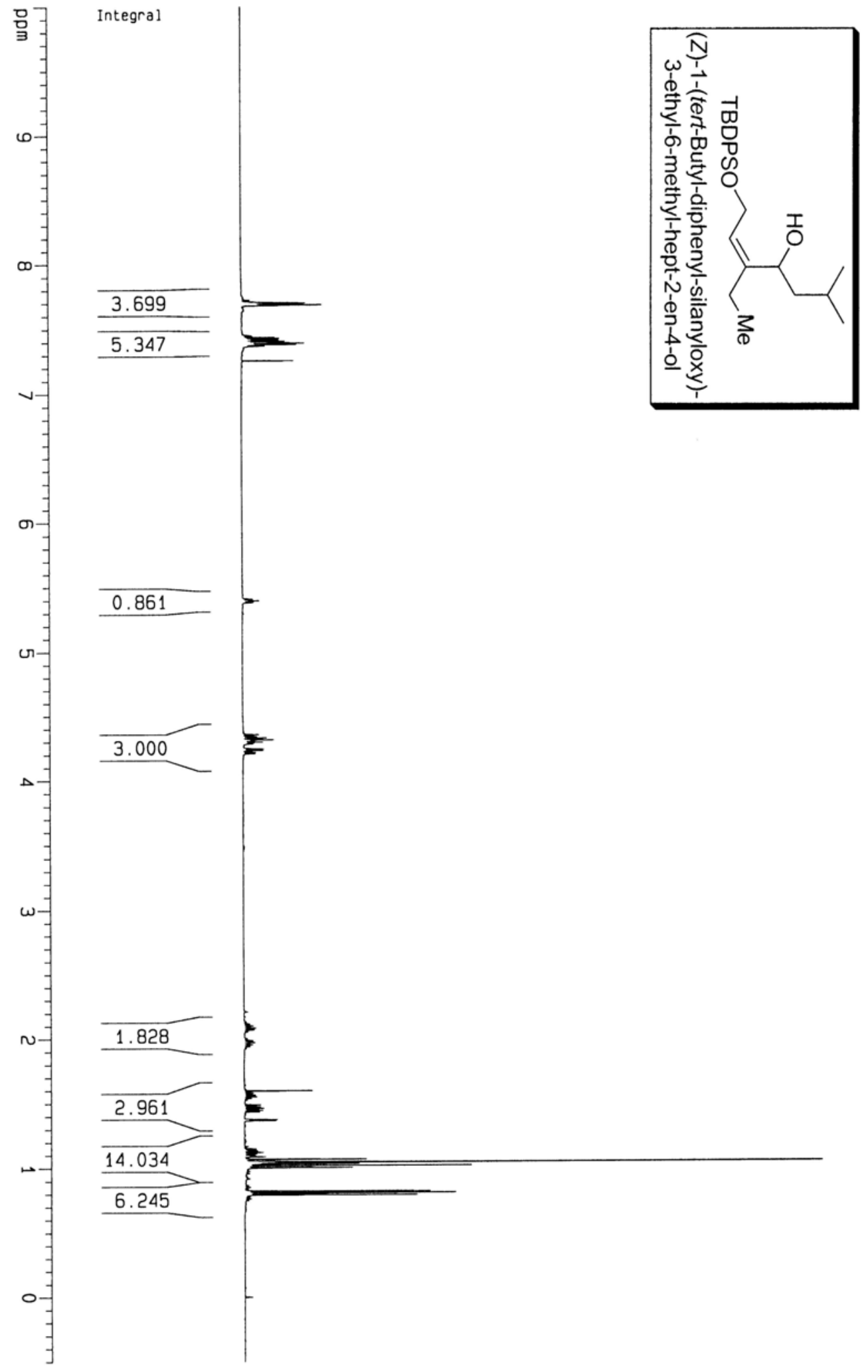




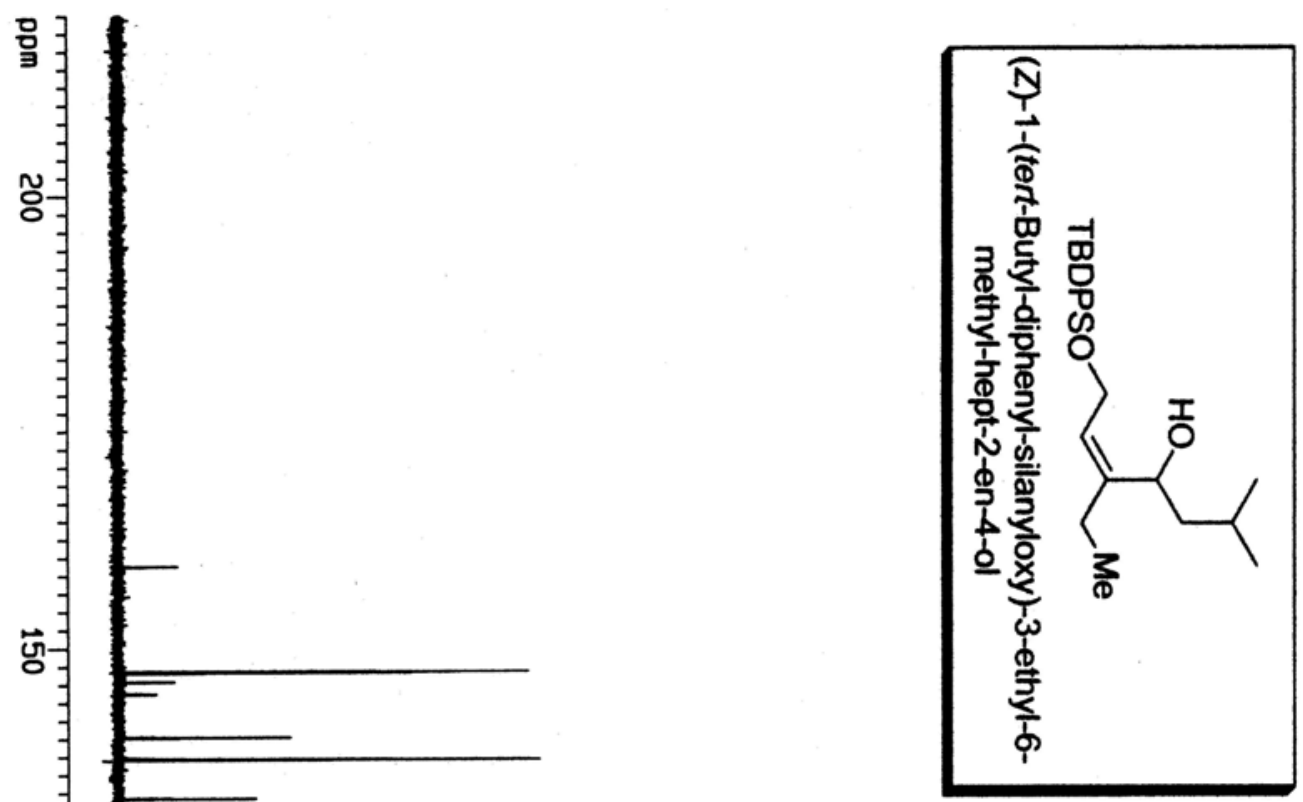




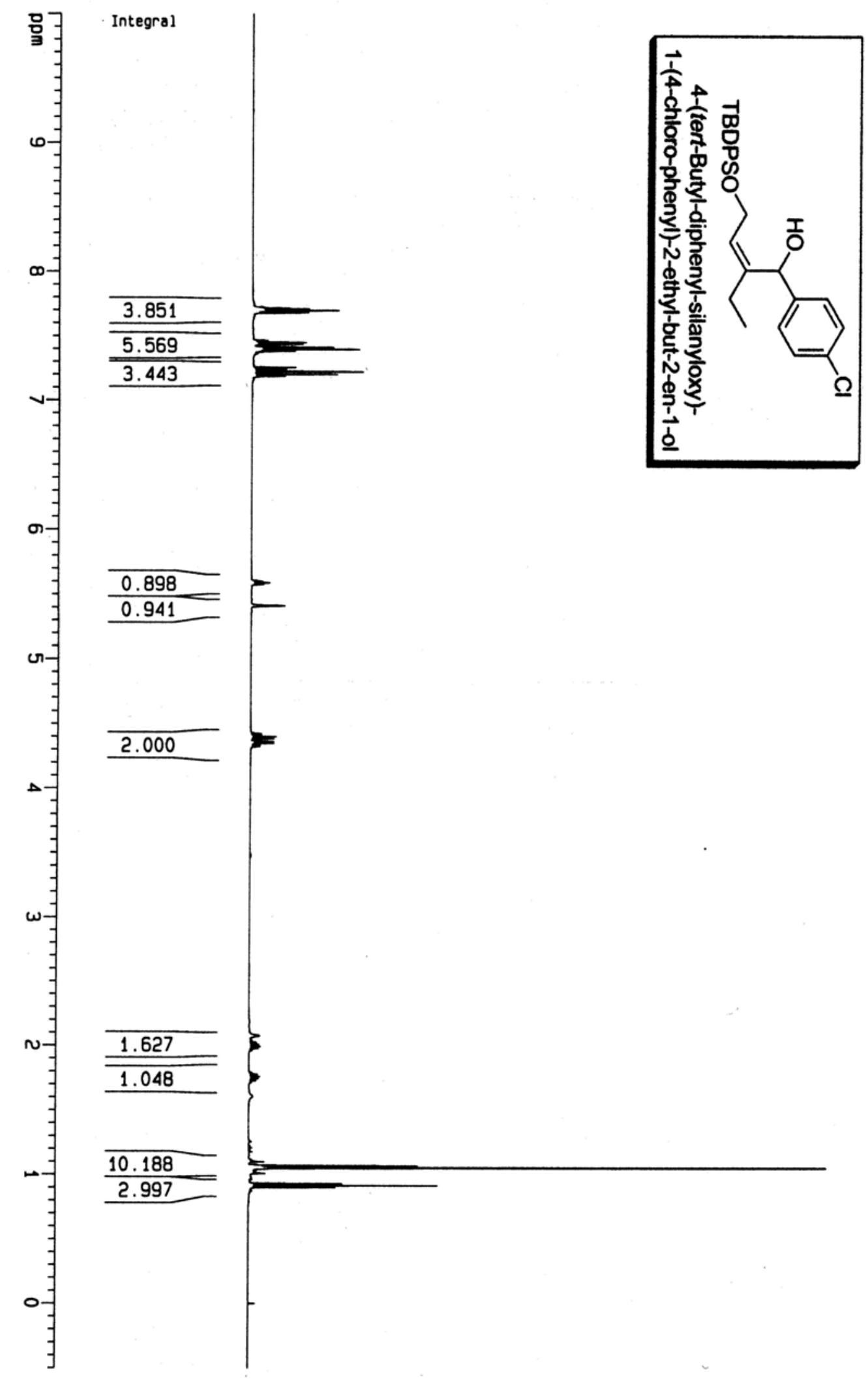




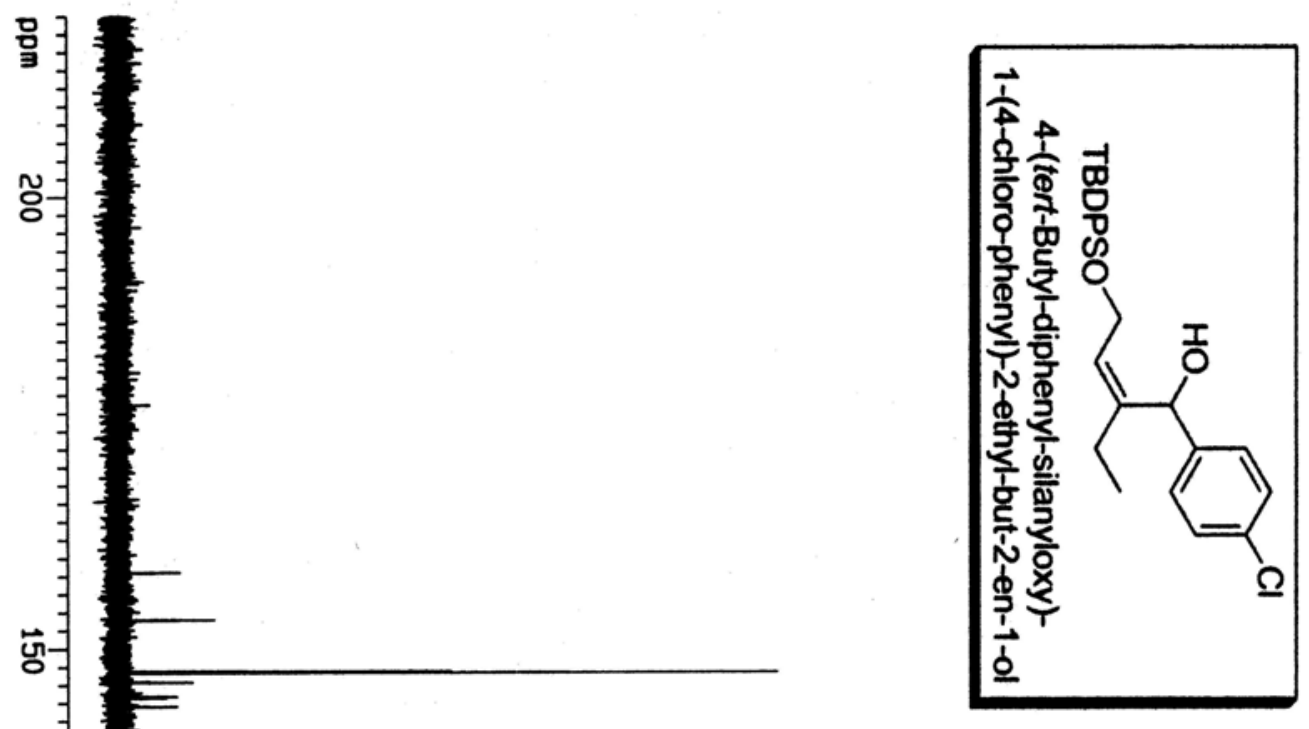



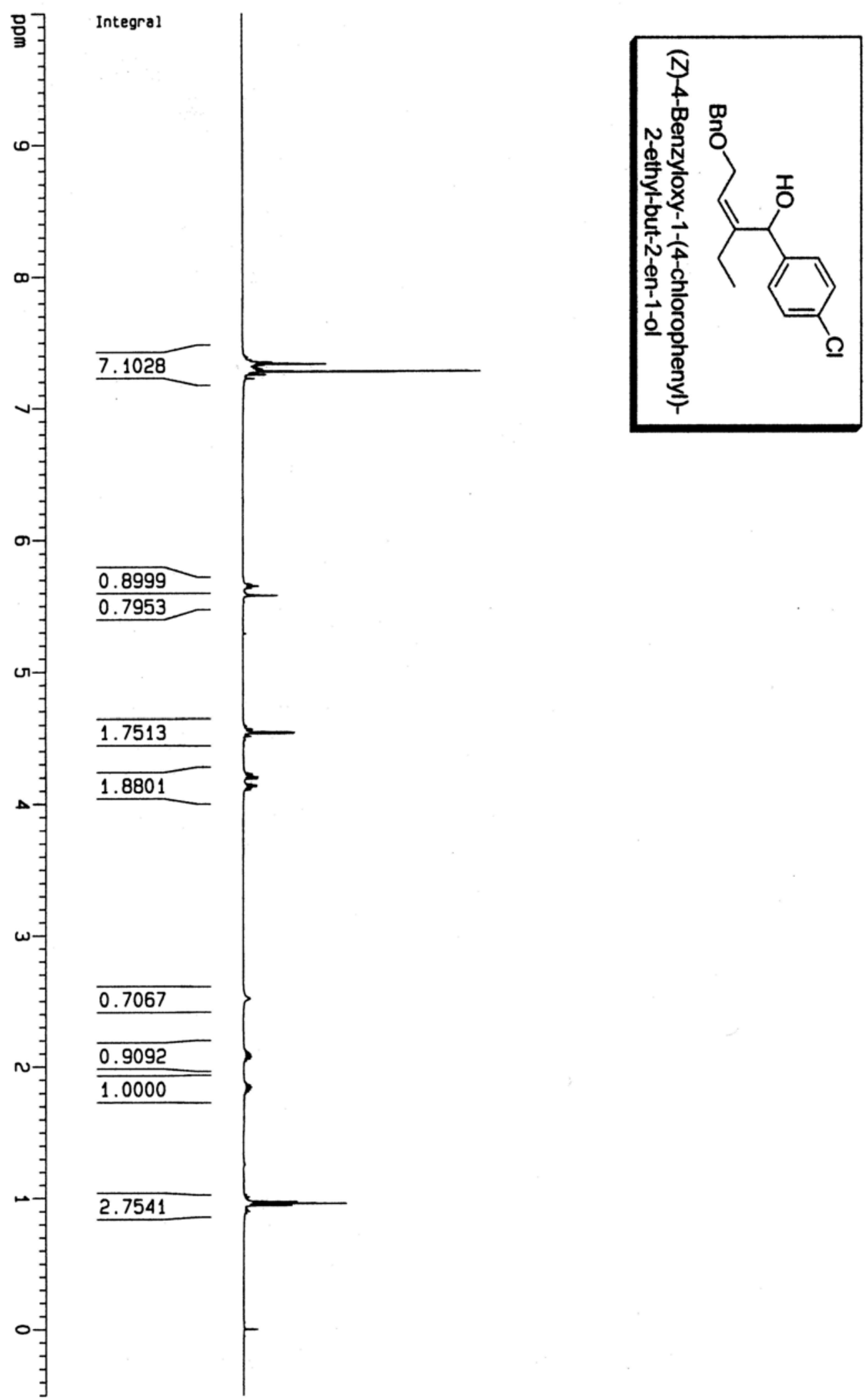

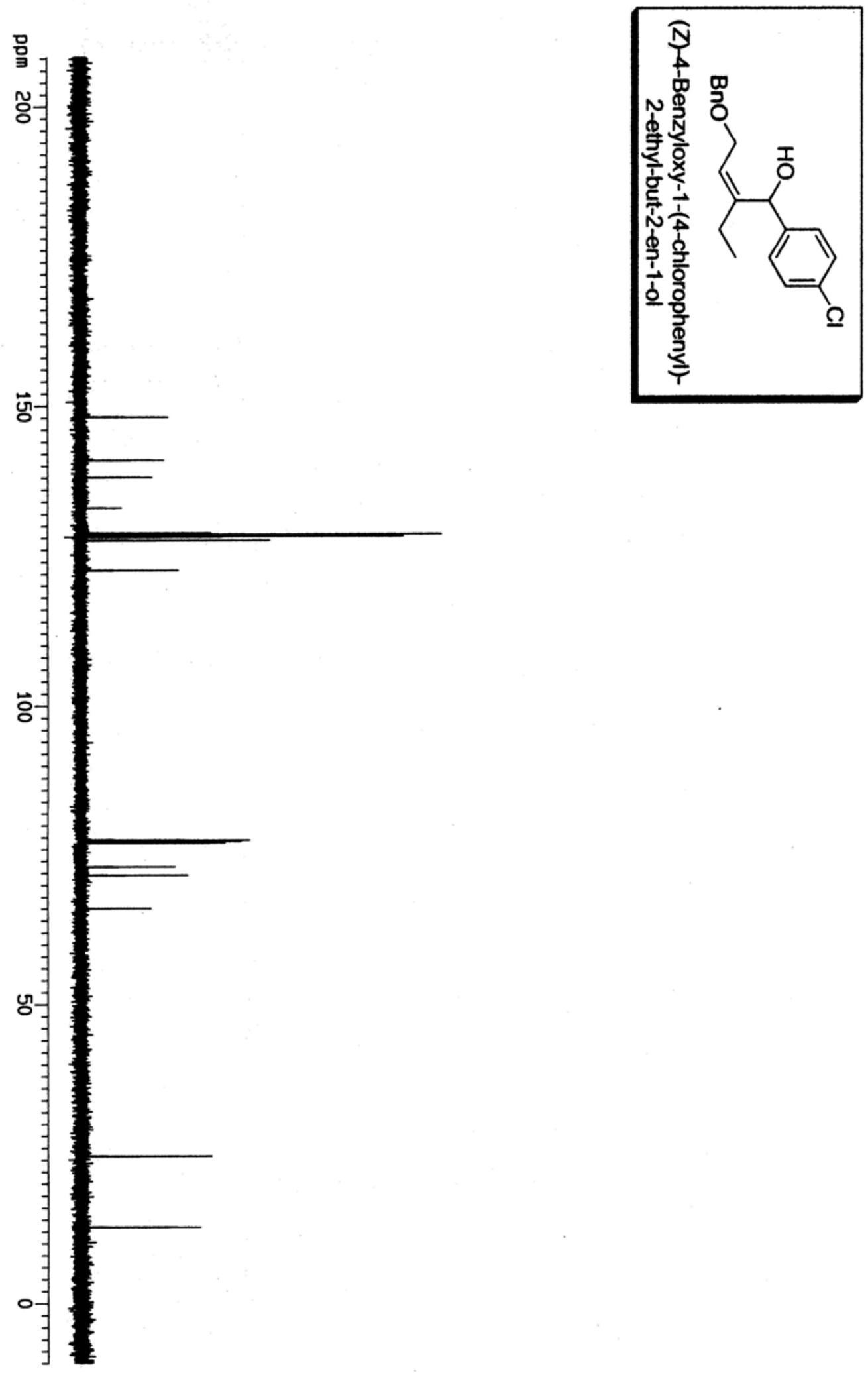


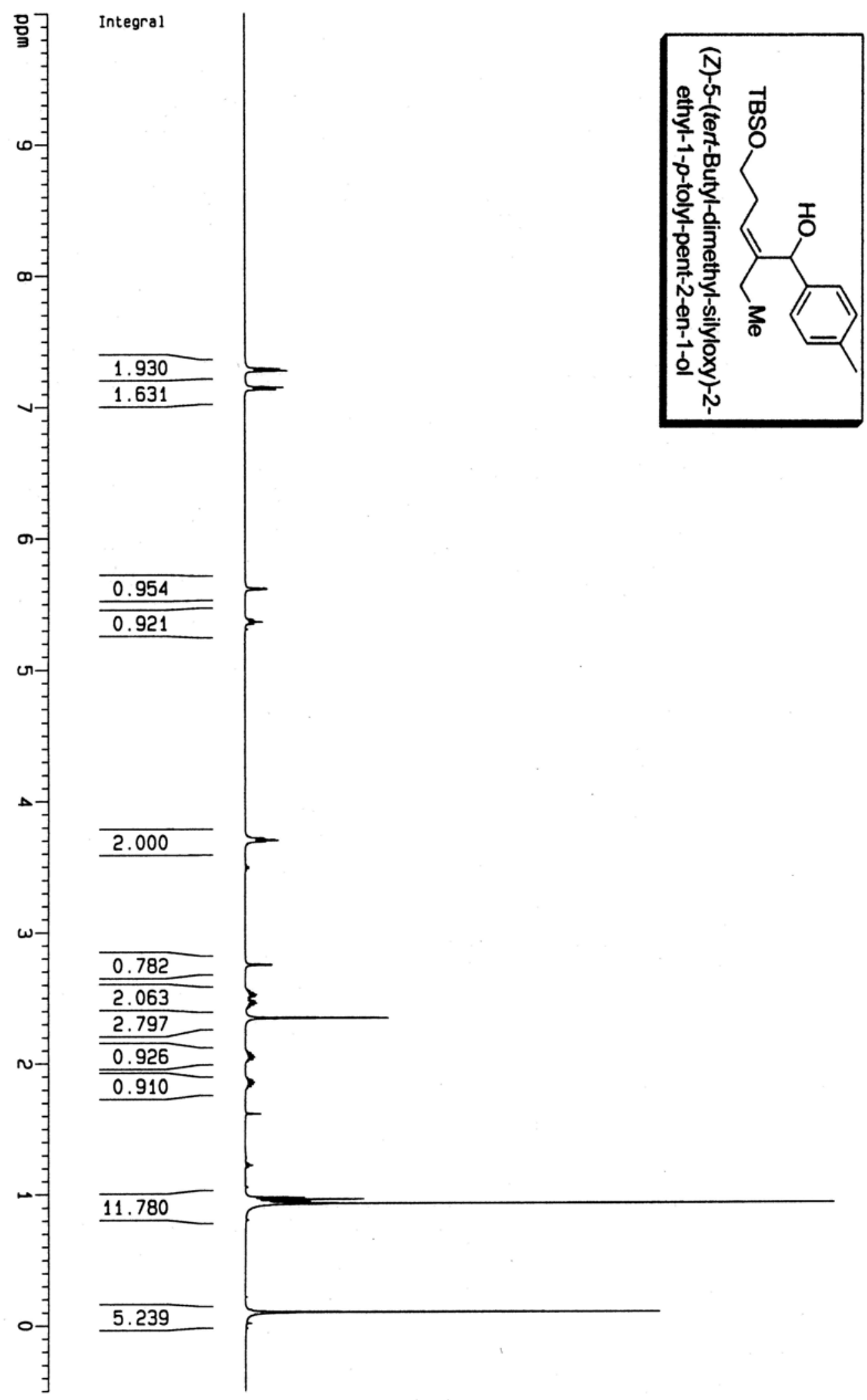



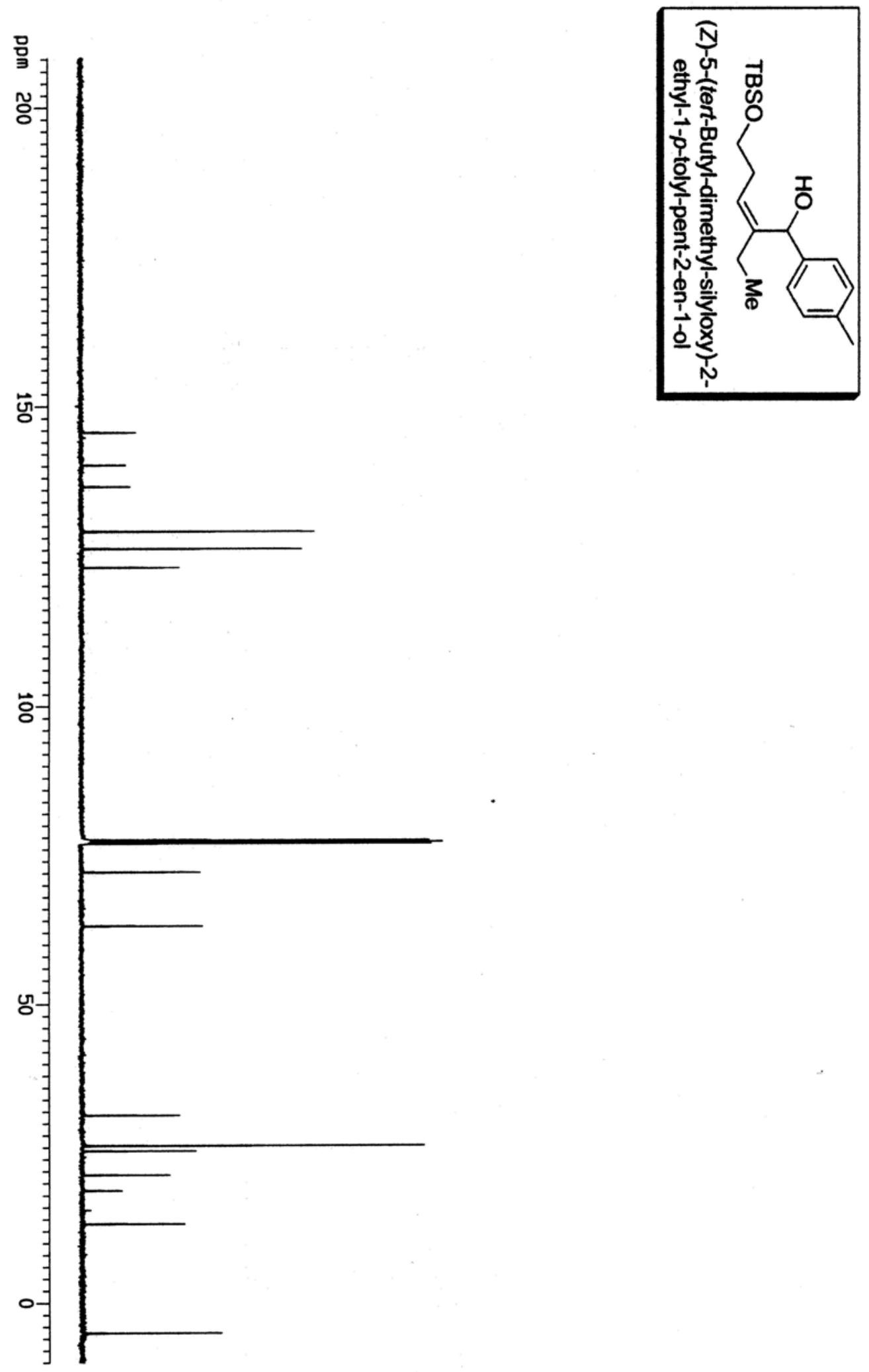


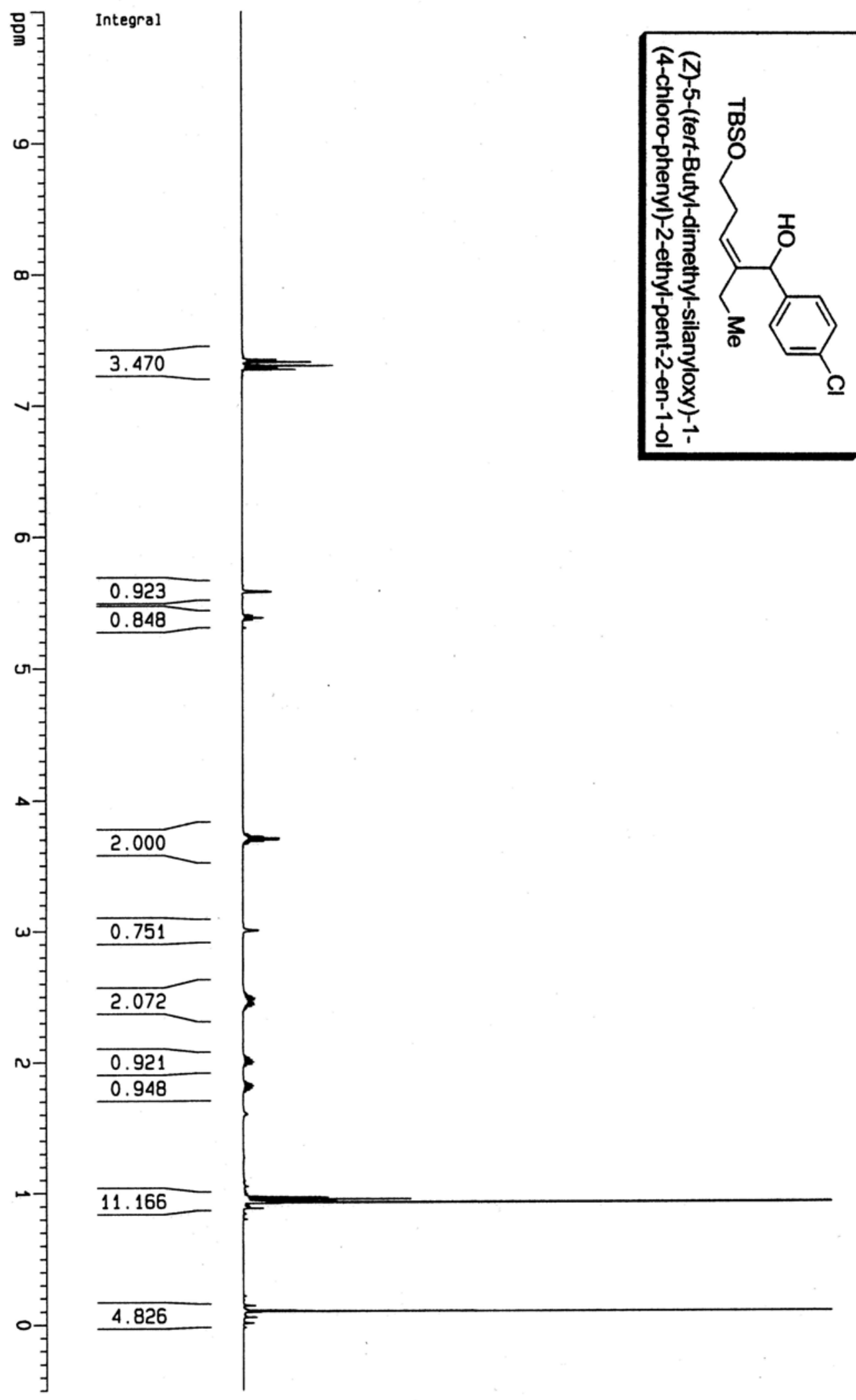



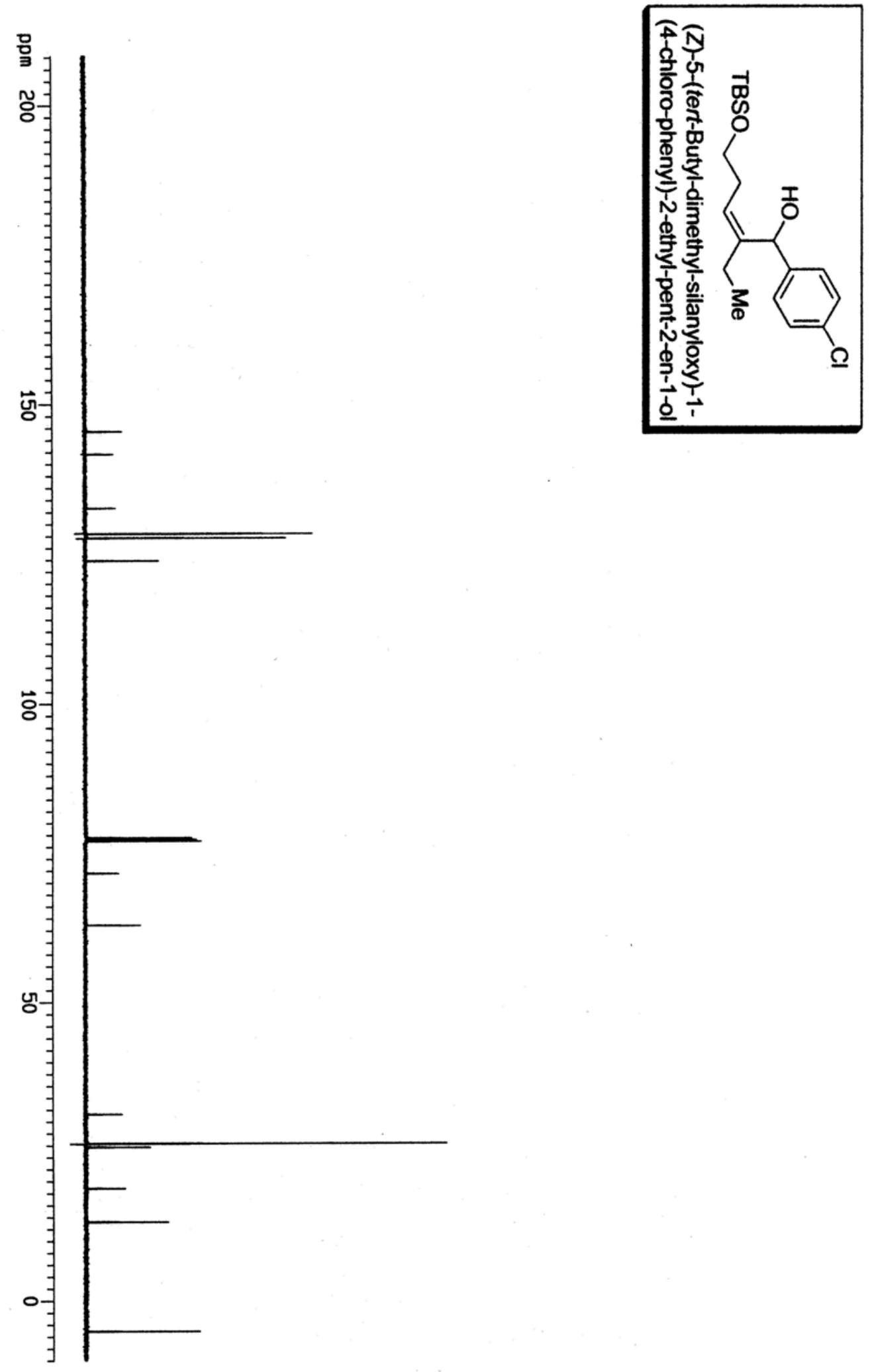

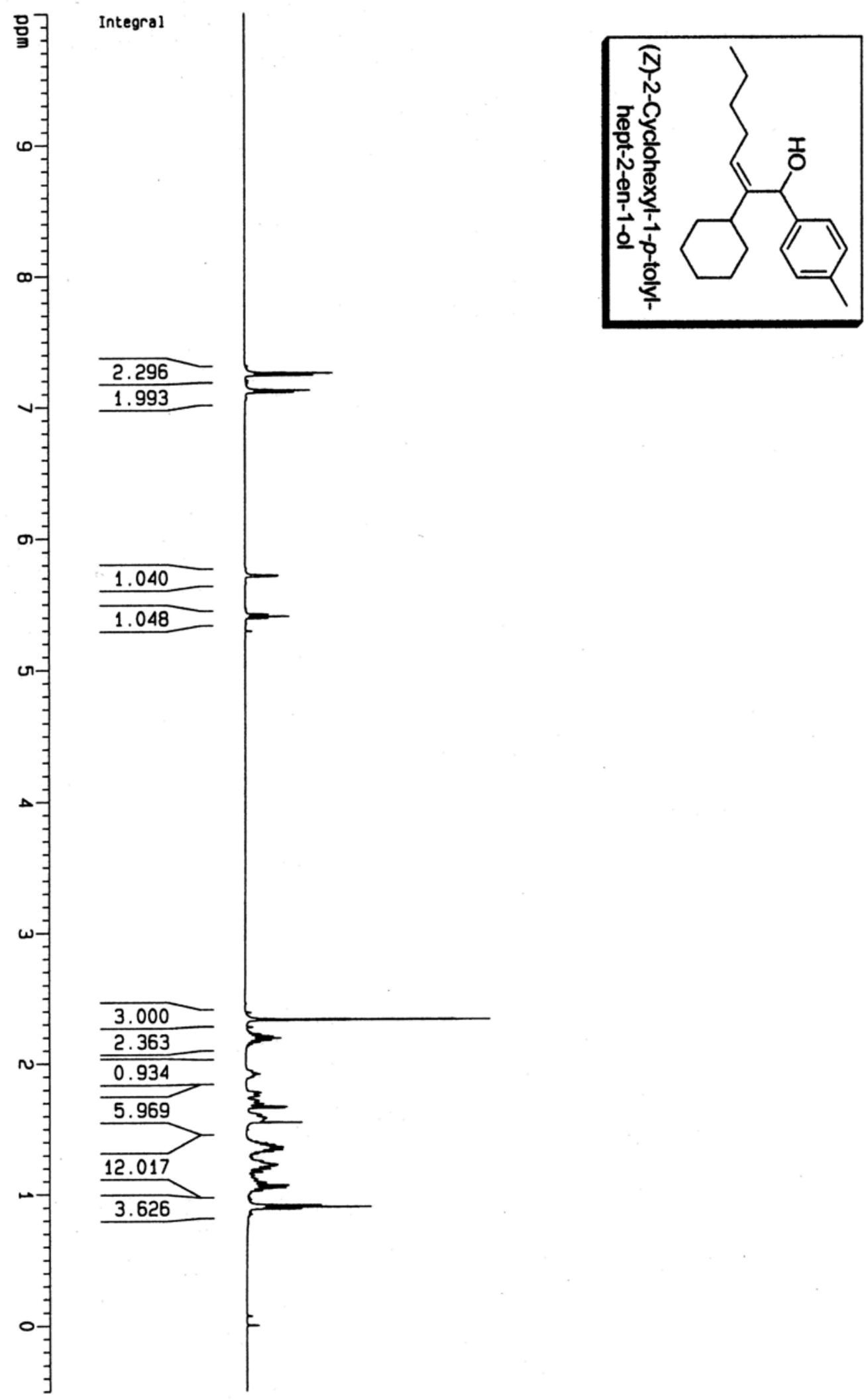

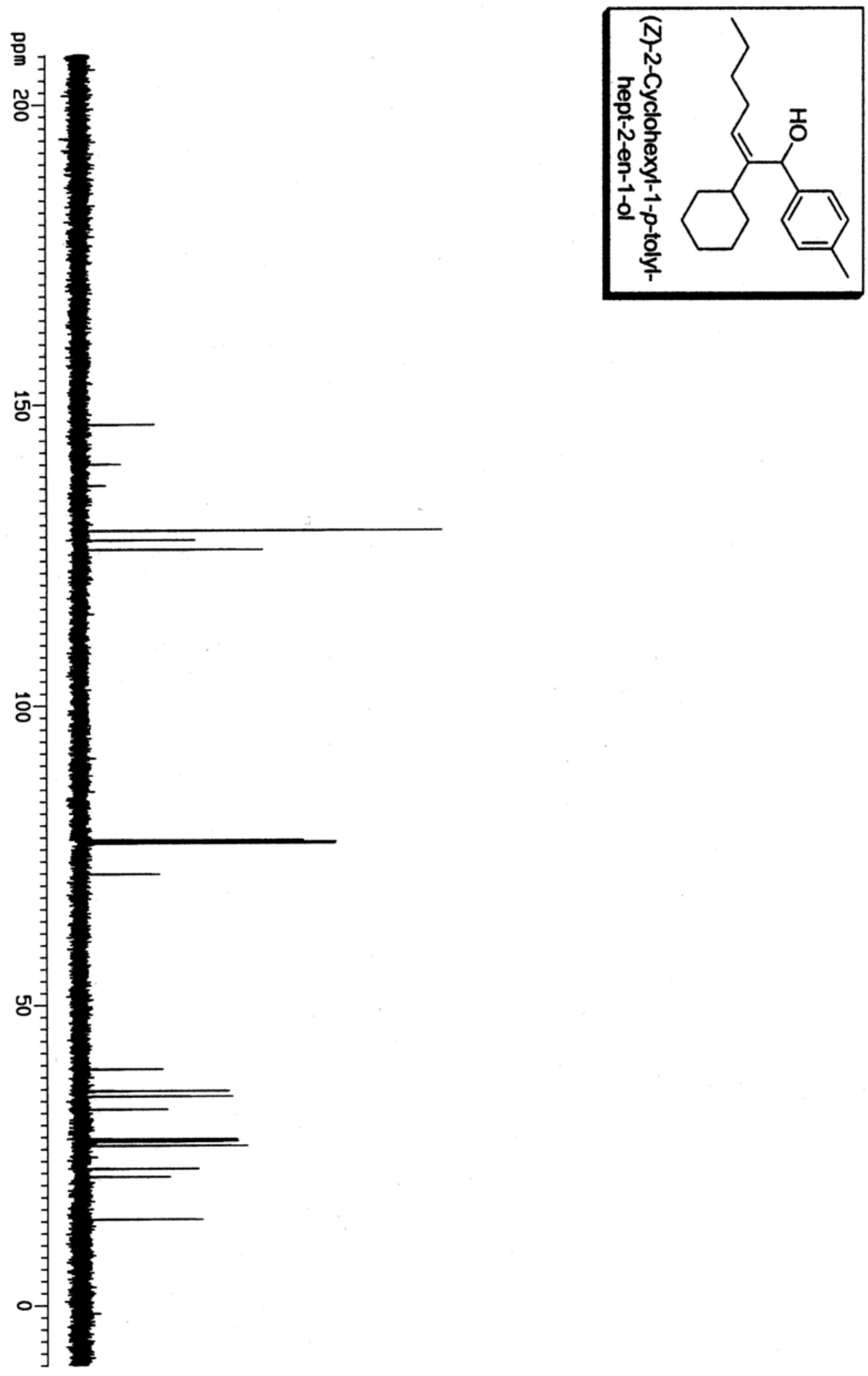


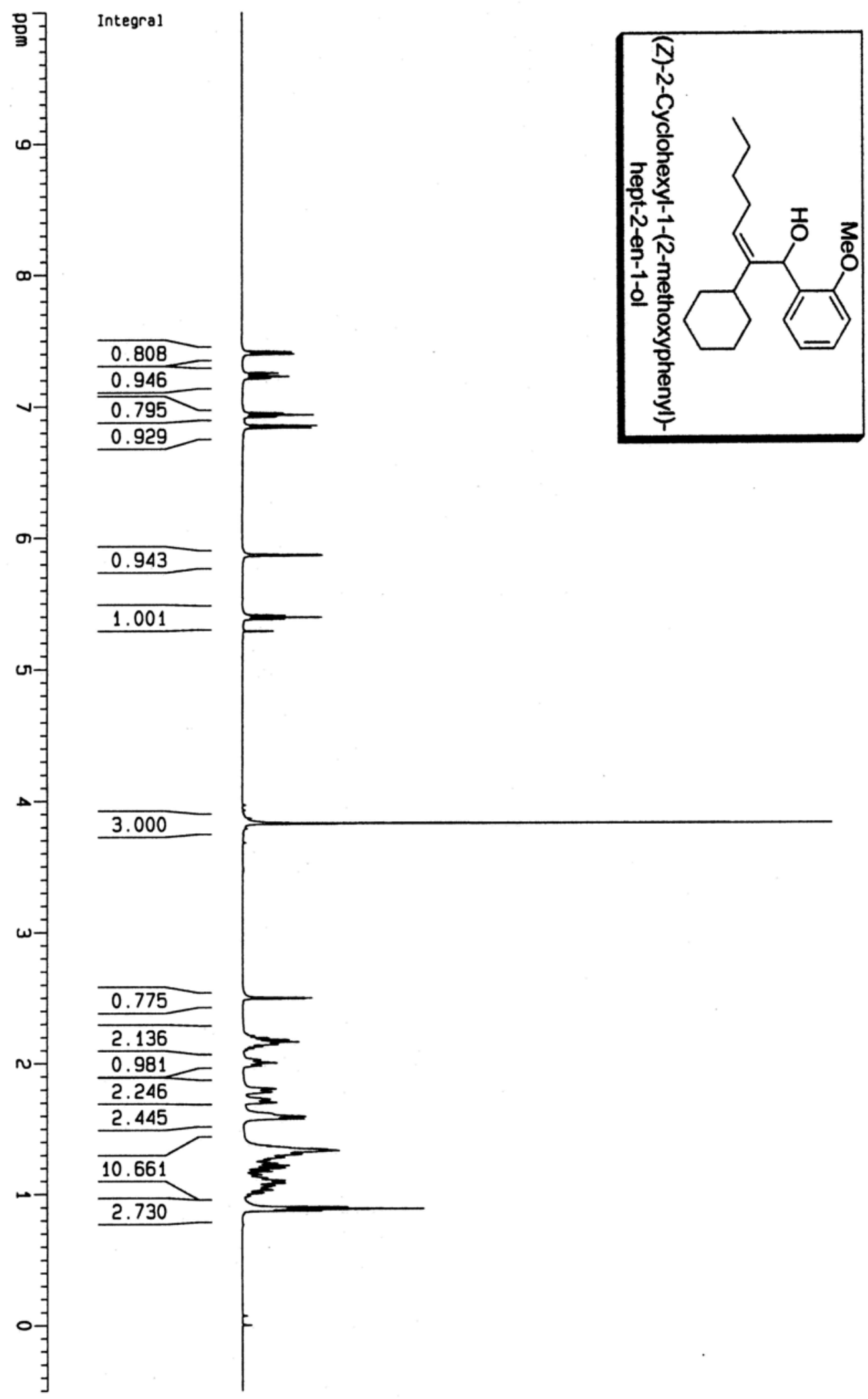




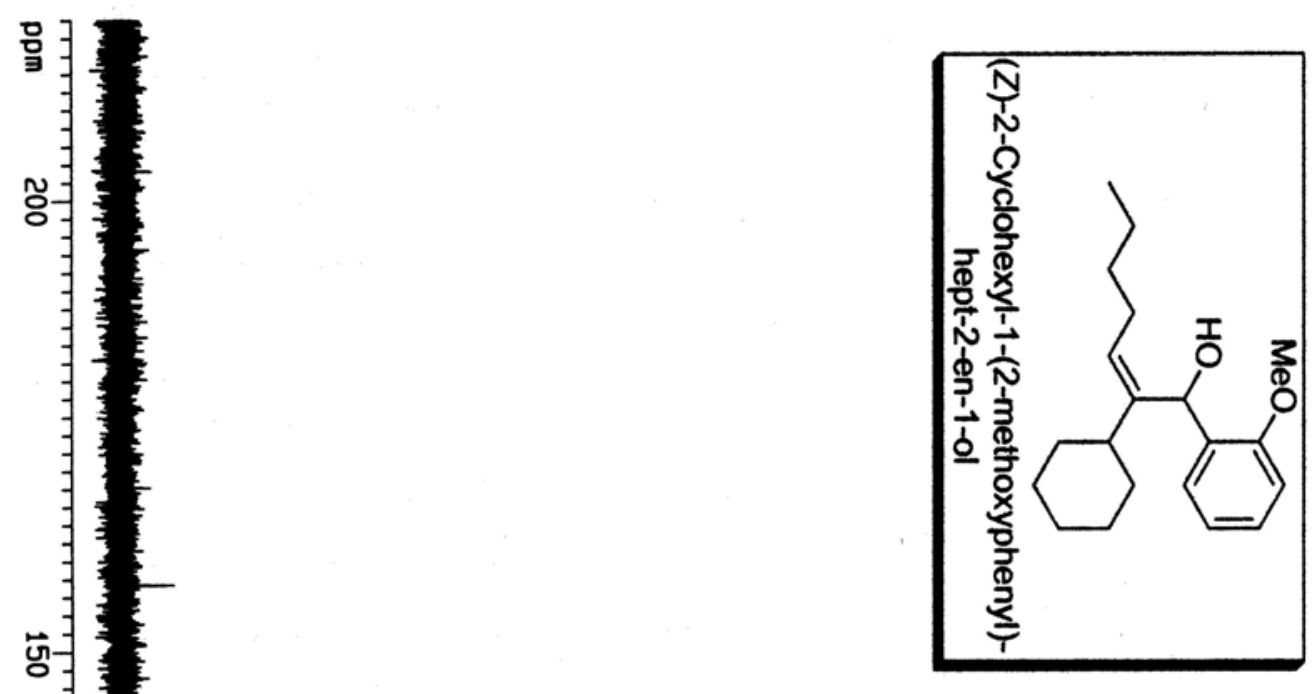




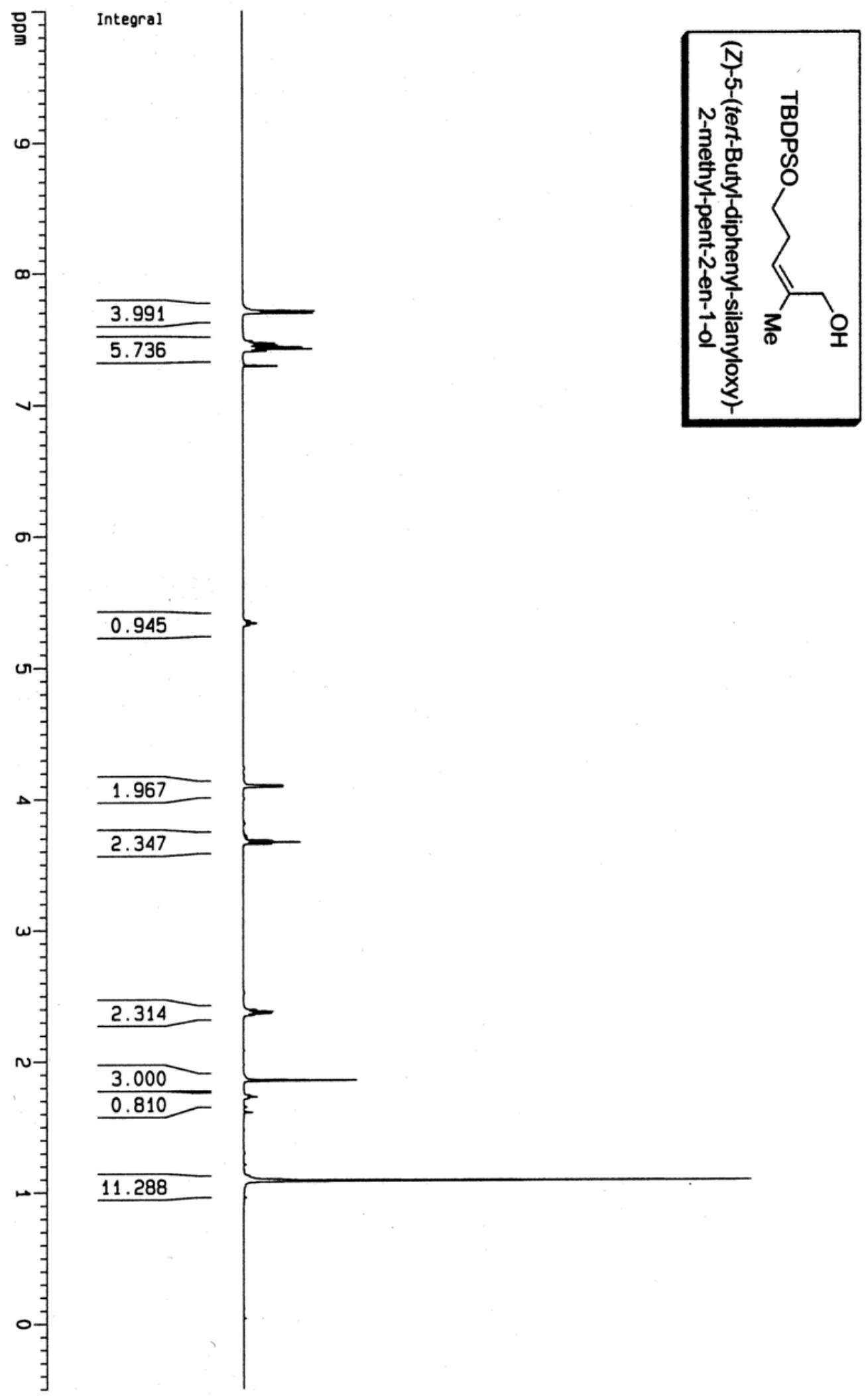




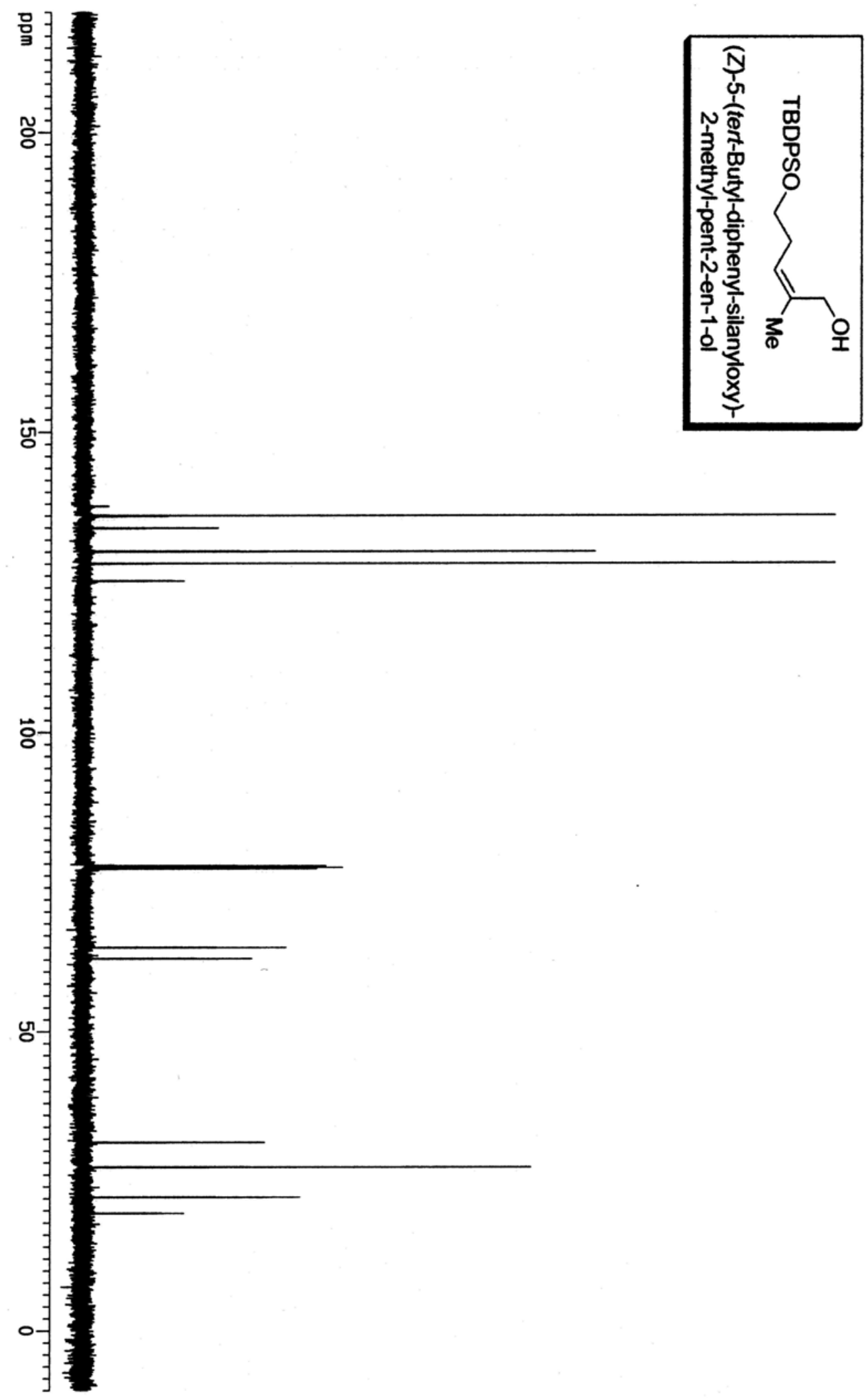




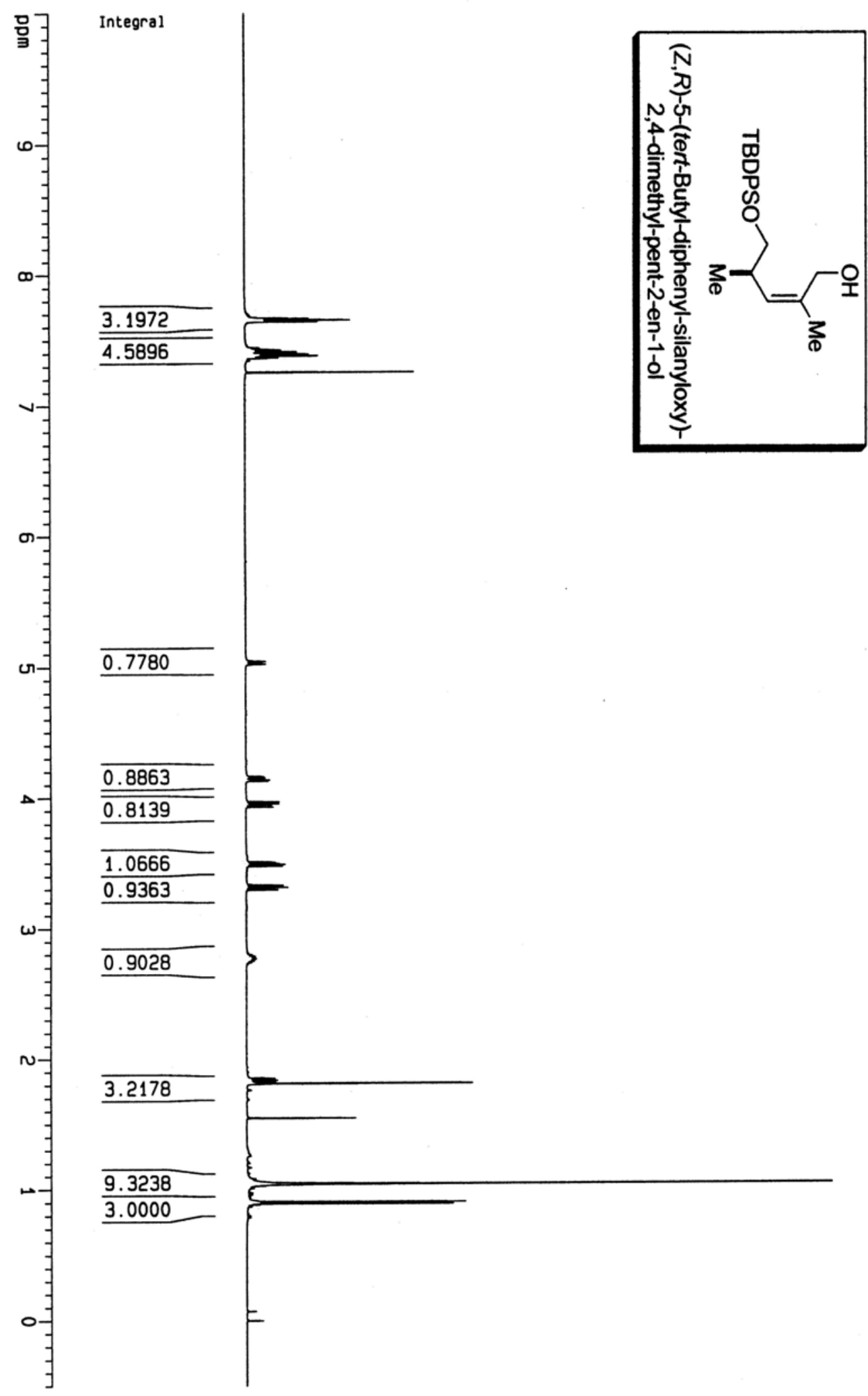




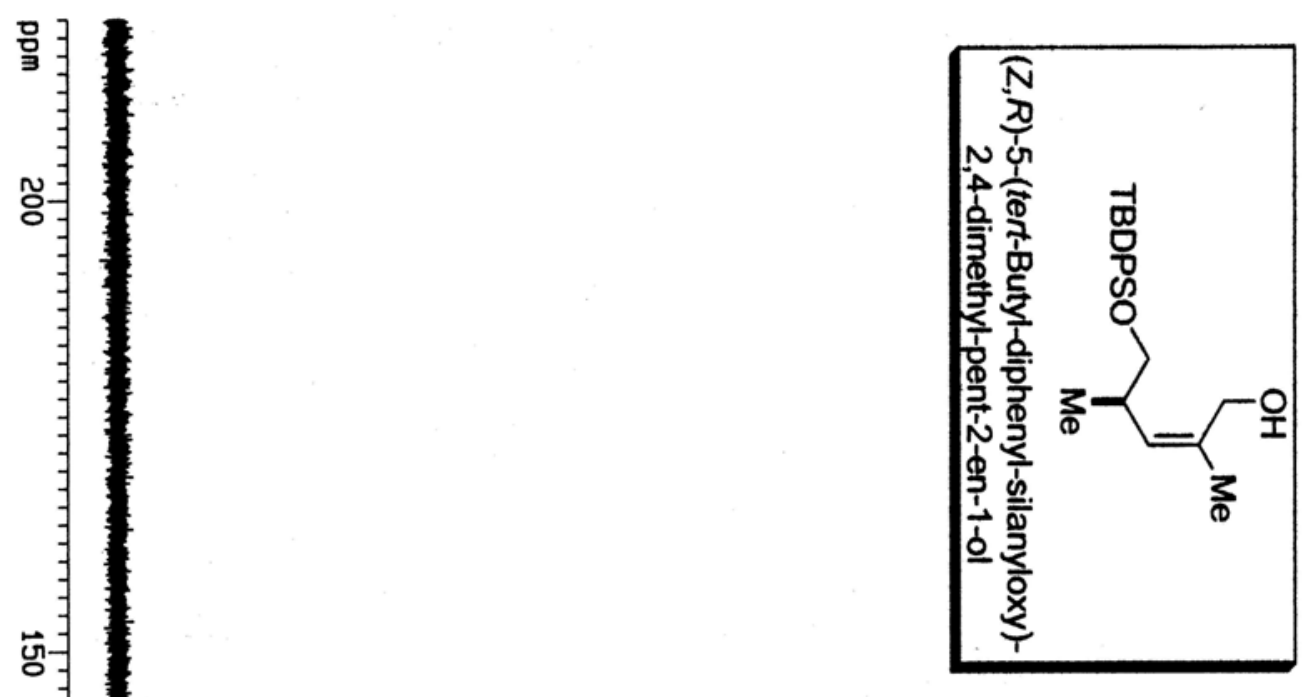



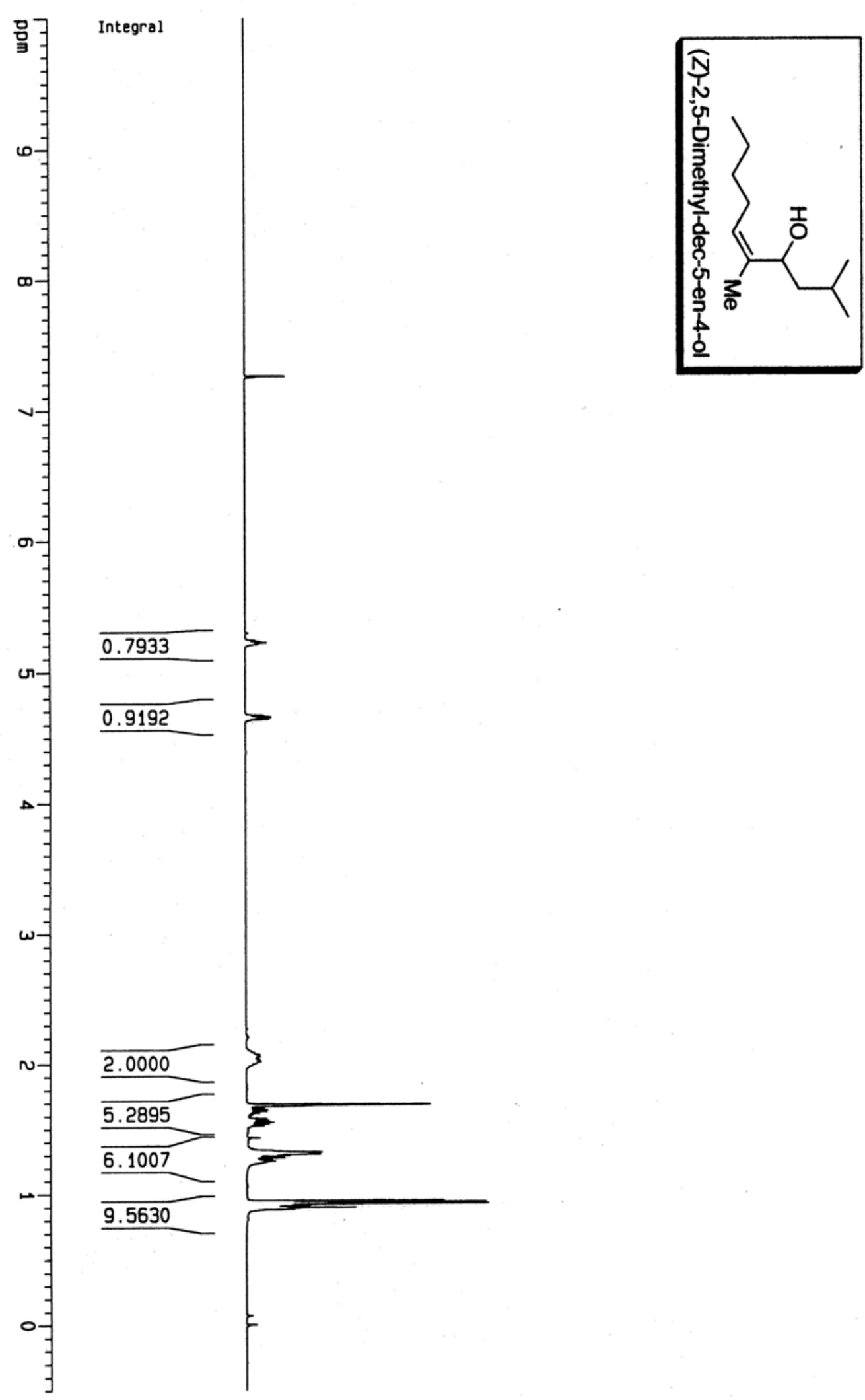

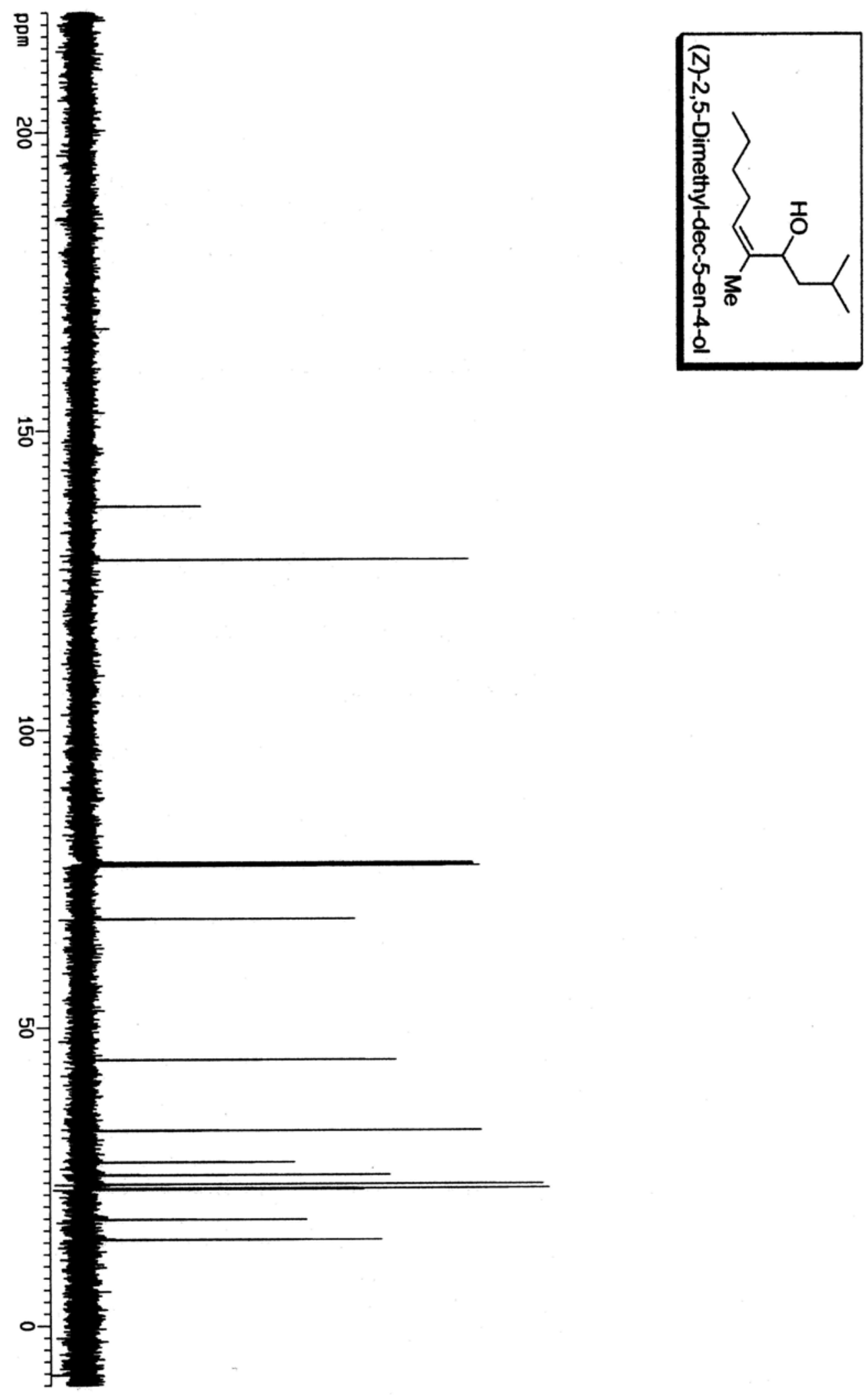

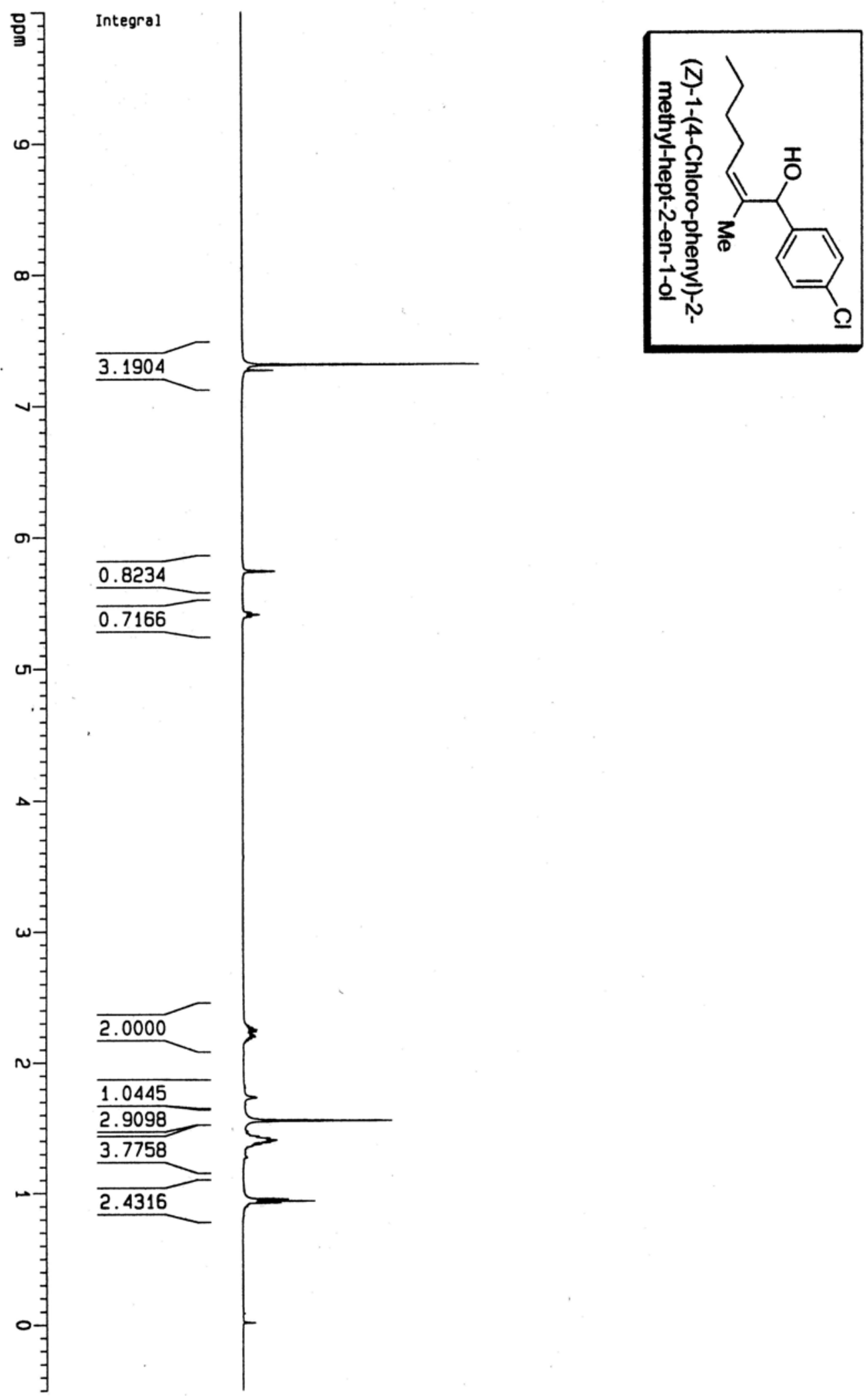

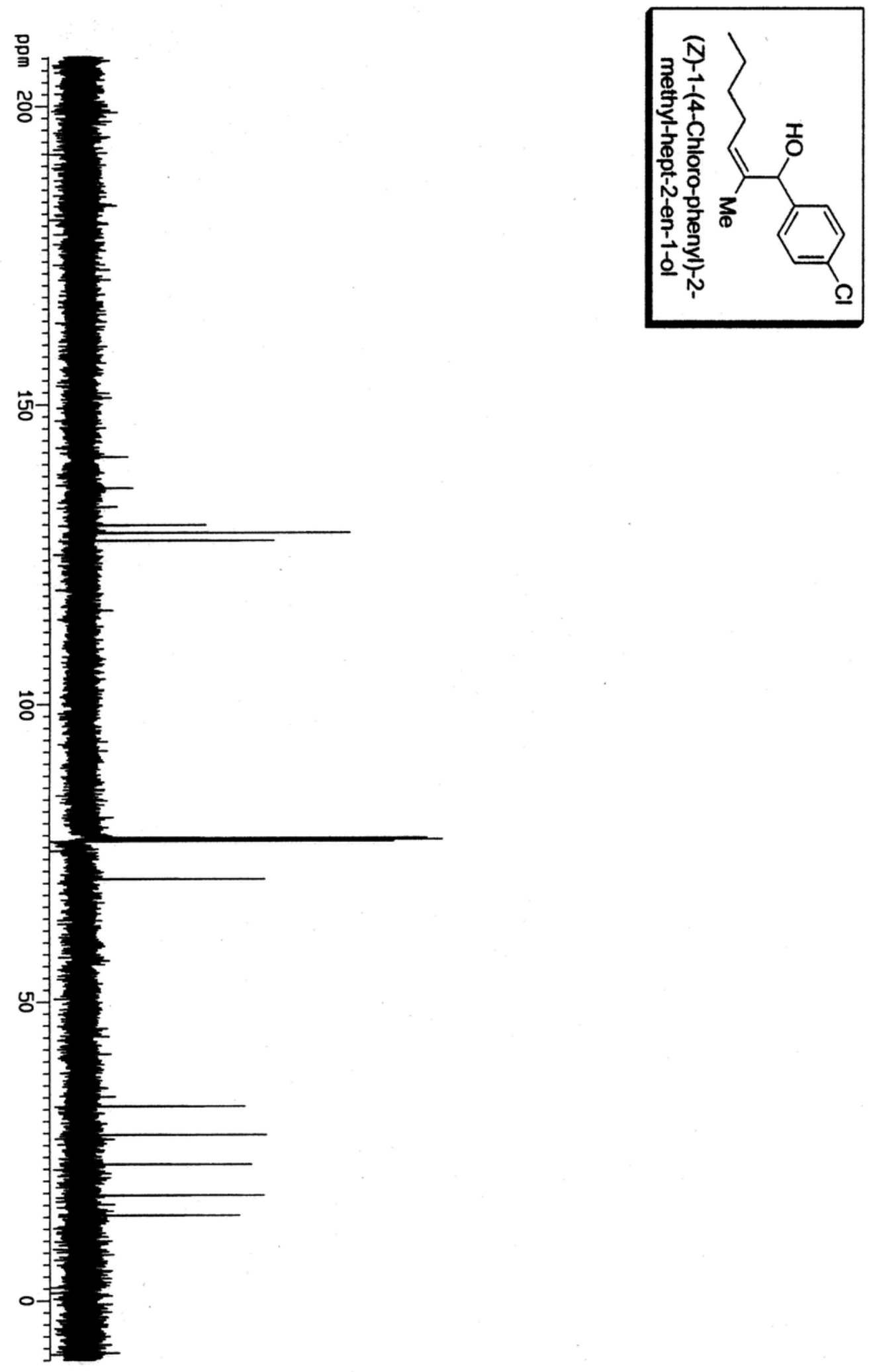

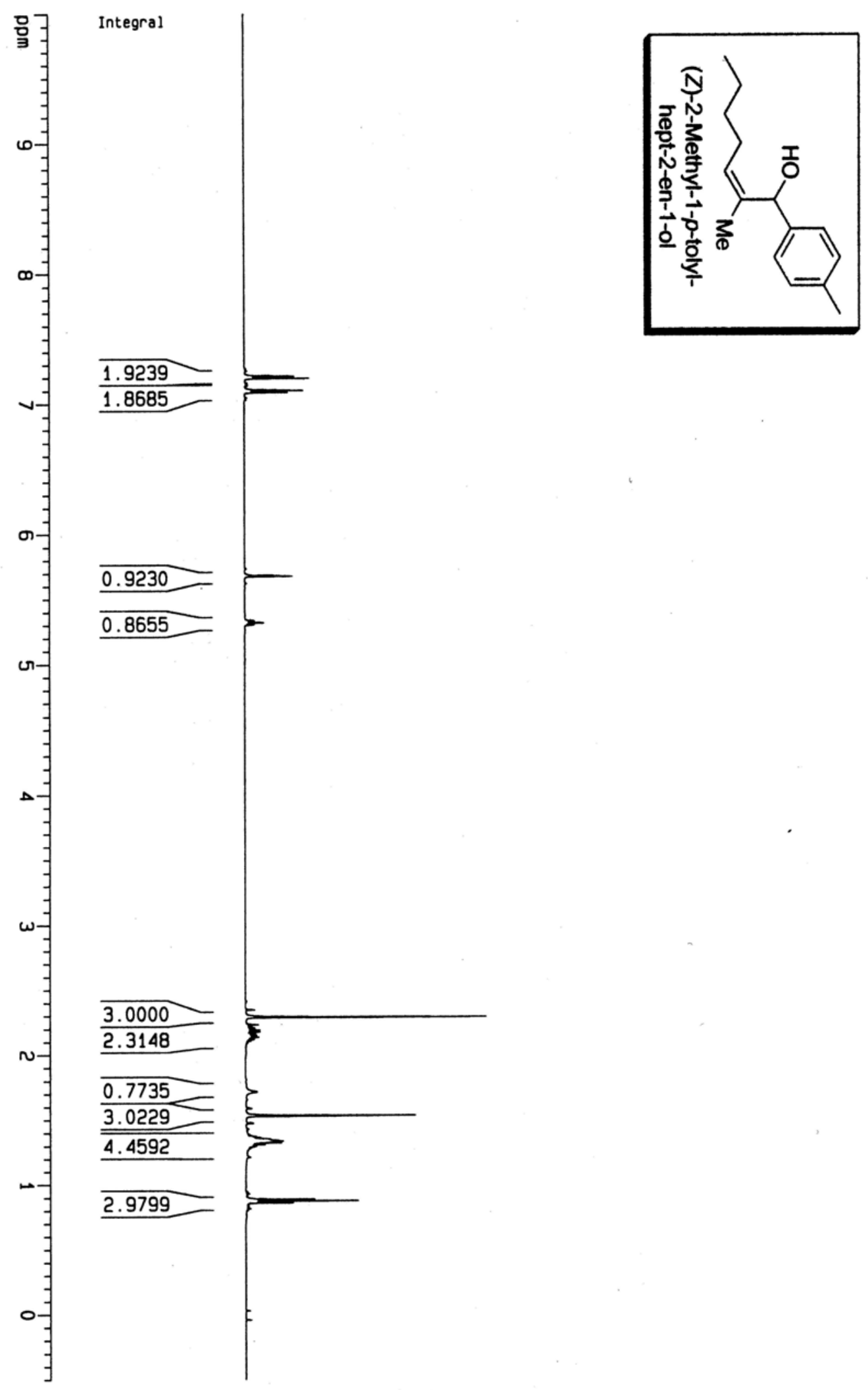

S43 

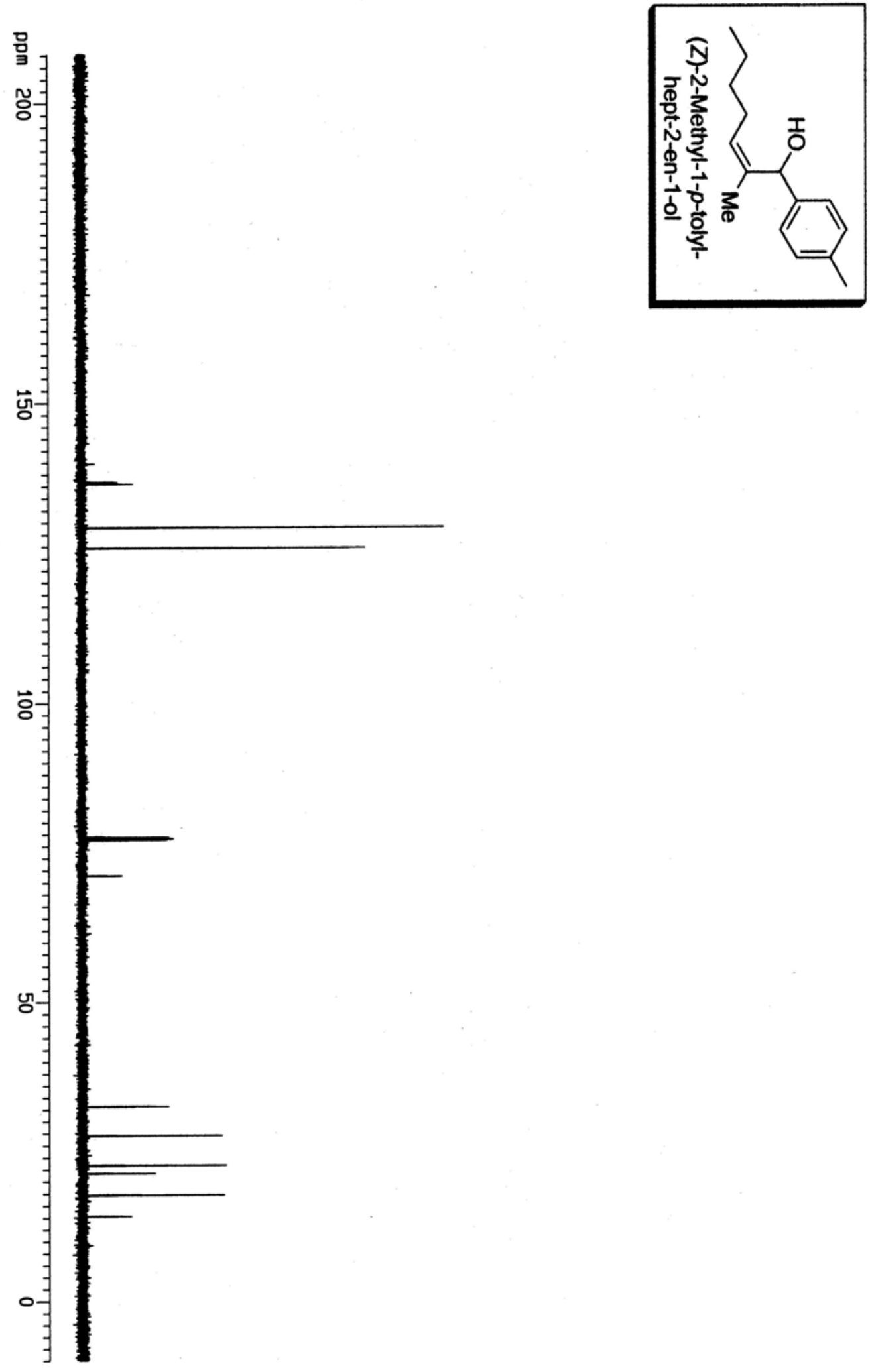


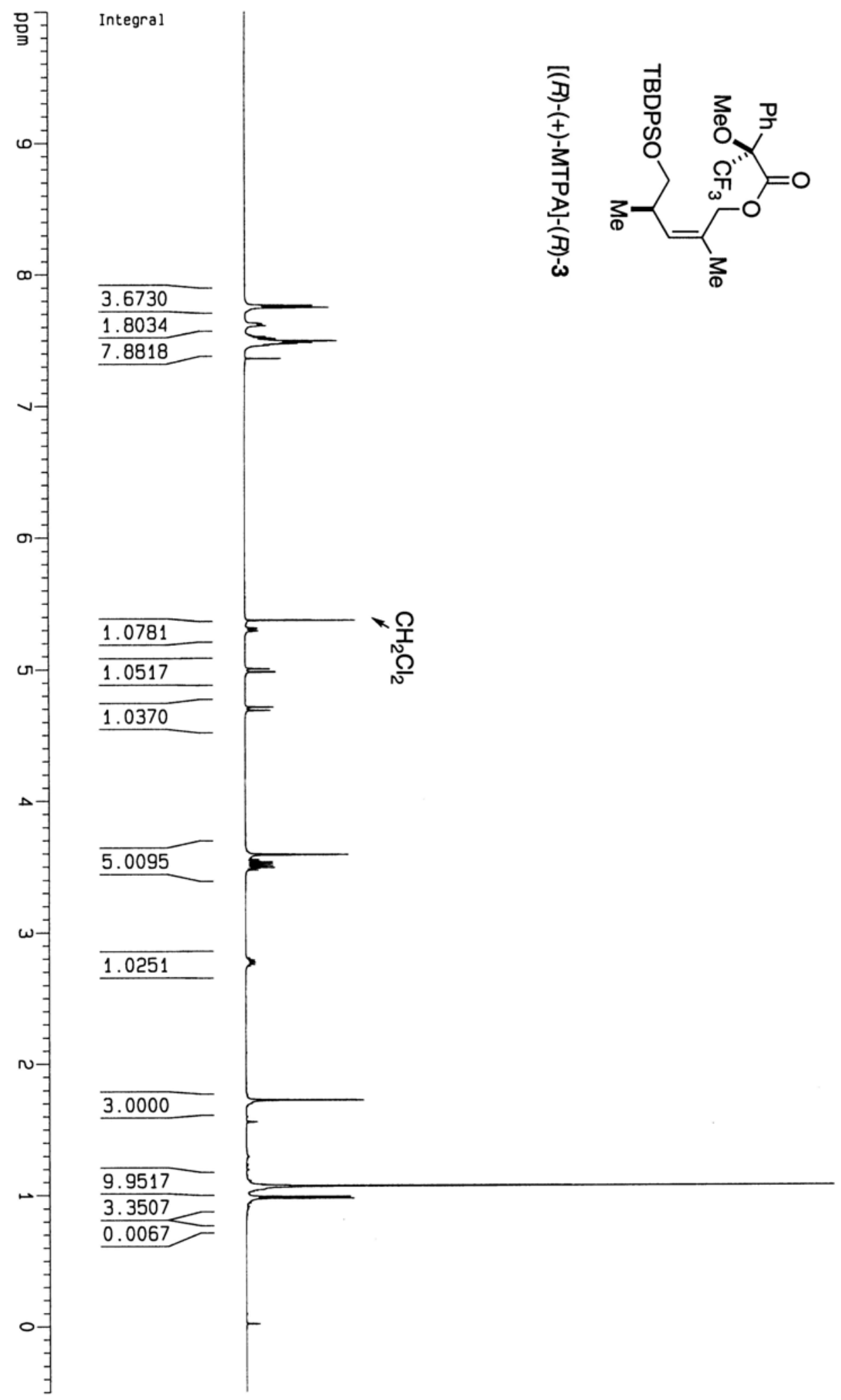




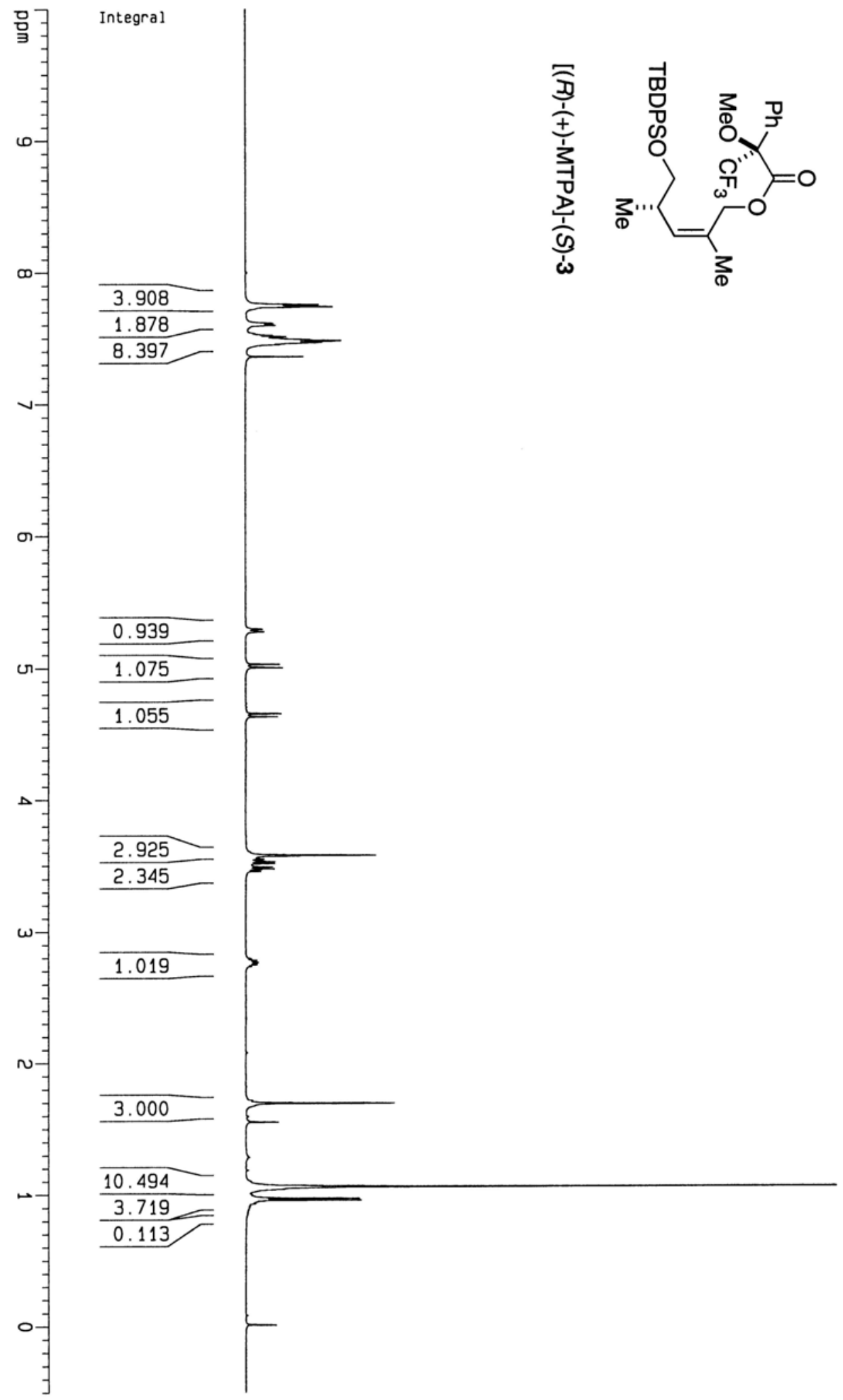

